asHOKa

UNIVERSITY

Ashoka University Economics

Discussion Paper 51

\title{
Generalized Perturbed Best Response Dynamics with a Continuum of Strategies
}

\section{February 2021}

Ratul Lahkar, Ashoka University

Sayan Mukherjee, Indian Statistical Institute, Kolkata

Souvik Roy, Indian Statistical Institute, Kolkata 


\title{
GENERALIZED PERTURBED BEST RESPONSE DYNAMICS
}

\section{WITH A CONTINUUM OF STRATEGIES}

\author{
Ratul Lahkar*, Sayan Mukherjee ${ }^{\dagger}$ and Souvik Roy ${ }^{\ddagger}$
}

February 9, 2021

\begin{abstract}
We consider a generalization of perturbed best response dynamics in population games with a continuum of strategies. The previous literature has considered the logit dynamic generated through the Shannon entropy as a deterministic perturbation. We consider a wider class of deterministic perturbations satisfying lower semicontinuity and strong convexity. Apart from the Shannon entropy, Tsallis entropy and Burg entropy are other perturbations that satisfy these conditions. We thereby generate the generalized perturbed best response dynamic with a continuum of strategies. We establish fundamental properties of the dynamic and show convergence in potential games and negative semidefinite games.
\end{abstract}

Keywords: Perturbed Best Response; Logit Dynamic; Potential Games; Negative Semidefinite Games.

JEL classification: C72; C73.

*Department of Economics, Ashoka University, Rajiv Gandhi Education City, Sonipat, Haryana, 131029, India. e-mail: ratul.lahkar@ashoka.edu.in

${ }^{\dagger}$ Economic Research Unit, Indian Statistical Institute, Kolkata. e-mail: sayanmukherjee012@gmail.com

$\ddagger$ (Corresponding Author) Economic Research Unit, Indian Statistical Institute, Kolkata. e-mail: souvik.2004@gmail.com 


\section{INTRODUCTION}

Perturbed best response dynamics are one of the most important classes of dynamics in evolutionary game theory. Best response is, of course, the fundamental behavioral norm in game theory. But due to multiplicity of best responses and the fact that the best response can change abruptly, the best response dynamic arising from this behavioral protocol is technically difficult to analyze (Gilboa and Matsui (1991), Hofbauer (1995)). Perturbed best response dynamics avoid this problem by considering a uniquely defined smoothed version of the best response which can then be analyzed using the standard theory of differential equations. The logit dynamic (Fudenberg and Levine (1998)) is the prototypical representative of this class of dynamics obtained by perturbing the best response through the Shannon entropy function. A number of authors have since generalized the logit dynamic to the class of perturbed best response dynamics (Benaim and Hirsch (1999), Hofbauer and Hopkins (2005), Hofbauer and Sandholm $(2002,2007)$ ).

As with most of evolutionary game theory, most of the literature on perturbed best response dynamics has been in the context of large population games with finite strategy sets. ${ }^{1}$ However, similar to classical game theory, various economic applications of evolutionary game theory are also more conveniently done with games with a continuum of strategies. Despite introducing certain measure theoretic complications related to, for example, defining the state space, continuous strategy models greatly simplify the characterization of Nash equilibrium and Pareto optimal states. ${ }^{2}$ Therefore, to make evolutionary game theory a more relevant technique for economic analysis, there is a need to develop this theory in the context of games with continuous strategy sets. This has been the main motivation behind the literature on extending the prominent evolutionary game dynamics from finite strategy to continuous strategy games. Papers in this area include Oechssler and Riedel $(2001,2002)$ and Cheung (2016) on the replicator dynamic, Hofbauer et al. (2009) on the Brown-von Neumann-Nash (BNN) dynamic, Cheung (2014) on the Smith dynamic and Perkins and Leslie (2014) and Lahkar and Riedel (2015) on the logit dynamic.

The present paper is a contribution to this literature on continuous strategy evolutionary dynamics. Specifically, it focuses on the class of perturbed best response dynamics for such games. The earlier literature on this topic has been confined to the logit dynamic (Perkins and Leslie (2014), Lahkar and Riedel (2015)). The logit dynamic is generated when agents' payoffs are perturbed using the Shannon entropy and agents best respond with respect to these perturbed

\footnotetext{
${ }^{1}$ See Sandholm (2010) for an extensive review of the literature on finite strategy evolutionary game theory.

${ }^{2}$ See, for example, Cheung and Lahkar (2018), Lahkar (2020) and Lahkar and Mukherjee $(2019,2021)$ for such applications to continuum strategy economic models like Cournot competition and implementation of efficiency in problems like public goods, public bads and the tragedy of the commons.
} 
payoffs. Here, we generalize the logit dynamic by considering perturbations other than the Shannon entropy. Thus, instead of assuming any particular form of perturbation, we consider a broader class of perturbations that satisfy certain lower semicontinuity and strong convexity conditions. For example, apart from the Shannon entropy, the Tsallis entropy and the Burg entropy are other admissible entropies under these conditions. As far as we know, this is the first paper that considers such a generalization of perturbed best response dynamics in continuous strategy games. We do note, though, that our generalisation is entirely with respect to deterministic perturbations unlike in the finite strategy literature where both deterministic and stochastic perturbations have been considered (Hofbauer and Sandholm (2002)). ${ }^{3}$

Similar to the logit best response, we first perturb an agent's payoff using the general perturbation function and allow the agent to best respond. This generates the generalized perturbed best response (GPBR) and the associated dynamic. We establish the fundamental properties of the dynamic. Thus, we show that rest points of the dynamic, which are fixed points of the perturbed best response function and which we call perturbed equilibria, exist and converge to Nash equilibria of the underlying game when the perturbation becomes small. We also show that unique solution trajectories that are continuous with respect to initial states exist for this class of dynamics. Results of a similar nature have, of course, already been established for the logit dynamic (Lahkar and Riedel (2015)). But by extending these results beyond the logit dyanamic, this paper establishes the idea of perturbed best response on sounder foundations in continuous strategy games.

This is important because one of main motivations behind looking for broader and more diverse classes of evolutionary dynamics is to ensure that the results of evolutionary game theory are not dependent upon the specific properties of a particular dynamic. Otherwise, those results will not be robust. Our findings are in line with this general quest in evolutionary game theory. Conclusions obtained from the application of the logit dynamic in continuous strategy games now become more credible since such results now generalize to the wider class of perturbed best response dynamics. This applies, for example, immediately to the results on evolutionary implementation in Lahkar and Mukherjee (2019) where the logit dynamic has been applied. These results now also hold for the entire class of deterministic perturbed best response dynamics.

Another ubiquitous pursuit in evolutionary game theory has been classes of games in which a wide variety of evolutionary dynamics converge to Nash equilibria. Nash equilibrium prediction in such games then becomes more credible even when agents are myopic as is the case in

\footnotetext{
${ }^{3}$ As far as we know, stochastic perturbations have not been studied in the context of continuous strategy games.
} 
evolutionary game theory. Two such classes of games in which almost all well known evolutionary dynamics, both finite strategy and continuous strategy, do converge are potential games (Monderer and Shapley (1996), Sandholm (2001)) and negative semidefinite games (Hofbauer and Sandholm (2009)). ${ }^{4}$ Potential games are defined by a real valued function which summarises payoffs in the game. Negative definite games are characterized by the property of "self-defeating externalities" due to which small groups of agents changing strategy find themselves at a relative payoff disadvantage. We show that our GPBR dynamic also converges in these two classes of games when their strategy sets are continuous. Convergence in this case is, of course, not to Nash equilibria but to their approximation, perturbed equilibria. Once again, such convergence have already been established for the continuous strategy logit dynamic. The present results show that such convergence arises from fundamental properties of perturbations like lower semicontinuity and convexity. This generalization is also in line with similar conclusions that arise in the case of finite strategy perturbed best response dynamics (Hofbauer and Sandholm (2007)).

Analyzing evolutionary dynamics for continuous strategy dynamics raises certain significant mathematical complications which makes the exercise a non-trivial extension of finite strategy dynamics. For example, an important question to resolve is the choice of topology in the space of population states, which takes a measure theoretic form. Following much of the literature (for example, Oechssler and Riedel (2001, 2002), Lahkar and Riedel (2015)) we apply the strong and weak topologies in our analysis. The weak topology is helpful in resolving questions like the existence of a perturbed equilibrium and convergence of solution trajectories of the perturbed best response dynamics to perturbed equilibria in potential and negative definite games. The strong topology, on the other hand, is required for results like the existence of solution trajectories of the dynamic.

Nor is the present analysis a straightforward extension of earlier papers on the continuous strategy logit dynamic. The absence of any specific functional form for the perturbation raises certain technical challenges that requires us make appropriate assumptions to make the analysis tractable. For example, we need assumptions like lower semicontinuity, strong convexity and the existence of a continuous density function for the perturbation to establish the existence of a perturbed equilibria. Showing convergence of perturbed equilibria to Nash equilibria requires us

\footnotetext{
${ }^{4}$ Monderer and Shapley (1996) originally introduced the notion of potential games in finite player games. Sandholm (2001) extended the concept to large population games with finite strategies. Further generalizations to large population games with continuous strategy sets can be found in, for example, Cheung (2014), Lahkar and Riedel (2015) and Cheung and Lahkar (2018). Negative semidefinite games are also called stable games (Hofbauer and Sandholm (2009)) and they generalize the fundamental idea of an evolutionary stable state (Maynard Smith (1982)). Continuous strategy extensions of such games have been considered in Hofbauer et al. (2009), Cheung (2014) and Lahkar and Riedel (2015).
} 
to impose some restrictions (Conditions A1 and A2) on the perturbation function to ensure that a small change in the population state does not lead to a large change in the perturbation. Such assumptions also ensure that, like the logit dynamic, our general class of perturbed best response dynamics are forward invariant not just in the state space, but in the space of states with bounded density functions. Such results then allow us to construct appropriate Lyapunov functions that establish convergence in the classes of potential and negative semidefinite games.

The rest of the paper is as follows. Section 2 defines preliminaries of the model. In Section 3, we analyze the perturbed best response and perturbed equilibria. This section also provides examples of perturbations beyond the Shannon entropy to which our analysis applies; namely, the Tsallis entropy and the Burg entropy. Section 4 establishes fundamental properties of the perturbed best response dynamic. In Sections 5 and 6, we show convergence in potential games and negative definite games respectively. Section 7 concludes.

\section{PRELIMINARIES}

We consider a population consisting of a continuum of agents of mass 1 . Let $S$ be a compact subset of a Polish ${ }^{5}$ space $X$ denoting the strategy space for each agent of the population. For simplicity, we can assume $S$ to be a compact interval in $\mathbb{R}$; results for general $S$ can be obtained in a similar manner. We denote the collection of all probability measures on $S$ by $\mathcal{P}(S)$ and the collection of all signed measures on $S$ by $\mathcal{M}(S)$. Let $\mathcal{M}_{0}(S)$ be the collection of all signed measures on $S$ whose measure of the whole space $S$ is 0 , that is, $\mathcal{M}_{0}(S):=\{\mu \in \mathcal{M}(S): \mu(S)=0\}$. We also use the notation $\mathcal{P}_{0}(S)$ to describe the set of all probability measures on $S$ that admit a probability density function or, equivalently, is absolutely continuous with respect to the Lebesgue measure. Finally, let $\mathcal{P}_{0}^{c}(S)$ be the set of probability measures which admits a continuous probability density function on $S$. We will denote the density of a generic probability measure $\mathbb{P}$ in $\mathcal{P}_{0}(S)$ by $p($.$) .$

A probability measure $\mathbb{P} \in \mathcal{P}(S)$ denotes the state of the population. This means that for any Borel set $A \in \mathcal{B}(S), \mathbb{P}(A)$ denotes the proportion of the population using strategies in $A$. Let $\mathcal{L}^{\infty}(S)$ be the set of bounded measurable functions on $S$ endowed with the sup-norm ${ }^{6}$ metric. We define a population game as a map $\pi: \mathcal{P}(S) \rightarrow \mathcal{L}^{\infty}(S)$ such that $\pi_{x}(\mathbb{P})$ is the payoff of an agent who plays strategy $x$ at population state $\mathbb{P}$. Throughout the paper, we assume that the population game $\pi$ is bounded in the sup-norm and weakly continuous with respect to the Prohorov metric. ${ }^{7}$

\footnotetext{
${ }^{5} \mathrm{~A}$ Polish space is a complete separable metric space.

${ }^{6}$ The sup-norm of $f \in \mathcal{L}^{\infty}(S)$ is defined as $\|f\|_{\infty}:=\sup _{x \in S}|f(x)|$.

${ }^{7}$ See (8) for the definition of Prohorov metric.
} 
A population state $\mathbb{P}^{\circ}$ is said to be a Nash equilibrium of the underlying population game $\pi$ if for all $x \in \operatorname{Supp}\left(\mathbb{P}^{\circ}\right)$ and all $y \in S$, we have $\pi_{x}\left(\mathbb{P}^{\circ}\right) \geq \pi_{y}\left(\mathbb{P}^{\circ}\right) .{ }^{8}$

We now seek to define the generalized deterministic perturbed best response dynamic. This in turn requires us to introduce the notions of a perturbed expected payoff and the perturbed best response. Suppose the current population state is $\mathbb{P} \in \mathcal{P}(S)$. We then define the expected payoff at $\mathbb{P}$ with respect to another probability measure $\mathbb{Q} \in \mathcal{P}(S)$ as $\mathbb{E}^{\mathbb{Q}}[\pi(\mathbb{P})]:=\int_{S} \pi(\mathbb{P}) d \mathbb{Q}$. We further define a deterministic perturbation function as a map $v: \mathcal{P}(S) \rightarrow \mathbb{R} \cup\{\infty\}$ with the restriction that $v(\mathbb{Q})=\infty$ if $\mathbb{Q} \notin \mathcal{P}_{0}(S)$. Let $\mathcal{L}^{1}(S)$ denote the collection of Lebesgue integrable functions on $S$ endowed with the $\|\cdot\|_{\mathcal{L}^{1}(S)}{ }^{9}$ norm. Also, define the following subset of $\mathcal{L}^{1}(S)$ :

$$
\mathcal{L}_{+}^{1,1}(S):=\left\{f \in \mathcal{L}^{1}(S) \mid f(x) \geq 0 \text { for all } x \in S \text { and } \int_{S} f(x) d x=1\right\}
$$

It is a well-known fact that for any $f \in \mathcal{L}_{+}^{1,1}(S)$, there exists a unique probability measure $\mathbb{Q}_{f} \in$ $\mathcal{P}_{0}(S)$ such that the probability density function of $\mathbb{Q}_{f}$ is $f$. We define the counterpart of $v$ as the mapping $\widetilde{v}: \mathcal{L}^{1}(S) \rightarrow \mathbb{R} \cup\{\infty\}$ such that

$$
\widetilde{v}(f)= \begin{cases}v\left(\mathbb{Q}_{f}\right) & \text { if } f \in \mathcal{L}_{+}^{1,1}(S) \\ \infty & \text { otherwise. }\end{cases}
$$

The fact that $\mathbb{Q}_{f}$ is unique for every $f \in \mathcal{L}_{+}^{1,1}(S)$ makes $\widetilde{v}$ well-defined. Throughout, we assume that $\widetilde{v}$ is lower semicontinuous ${ }^{10}$ and strongly convex ${ }^{11}$ with respect to the $\|\cdot\|_{\mathcal{L}^{1}(S)}$ norm. One interpretation of $\mathbb{Q}$ is that it is a mixed strategy and $\mathbb{E}^{\mathbb{Q}}[\pi(\mathbb{P})]$ is the expected payoff of an agent who plays strategy $\mathbb{Q}$ at the population state $\mathbb{P}$. The perturbed expected payoff for the agent with respect to $v$ is then $\mathbb{E}^{\mathbb{Q}}[\pi(\mathbb{P})]-v(\mathbb{Q})$. Given $\eta>0$, we define the generalized perturbed best

\footnotetext{
${ }^{8}$ We denote the support of a probability measure $\mathbb{P}$ by $\operatorname{Supp}(\mathbb{P})$.

${ }^{9}$ The $\|\cdot\|_{\mathcal{L}^{1}(S)}$ norm of $f \in \mathcal{L}^{1}(S)$ is $\|f\|_{\mathcal{L}^{1}(S)}:=\int_{S}|f(x)| d x$.

${ }^{10}$ A mapping $h: \mathcal{L}^{1}(S) \rightarrow \mathbb{R} \cup\{\infty\}$ is said to be lower semicontinuous with respect to the $\|\cdot\|_{\mathcal{L}^{1}(S)}$ norm if for all sequences $\left(f_{n}\right)_{n \geq 1}$ in $\mathcal{L}^{1}(S),\left\|f_{n}-f\right\|_{\mathcal{L}^{1}(S)} \rightarrow 0$ implies

$$
\liminf _{n \rightarrow \infty} h\left(f_{n}\right) \geq h(f) .
$$

${ }^{11}$ An extended function $T$ on $\left(\mathcal{L}^{1}(S),\|\cdot\|_{\mathcal{L}^{1}(S)}\right)$ to $\mathbb{R} \cup\{\infty,-\infty\}$ is said to be strongly convex if there exists $L>0$ such that

$$
T(\alpha f+(1-\alpha) g) \leq \alpha T(f)+(1-\alpha) T(g)-\frac{L \alpha(1-\alpha)}{2}\|f-g\|_{\mathcal{L}^{1}(S)}^{2}
$$

for all $f, g \in \operatorname{dom} T$ and $\alpha \in[0,1]$ where $\operatorname{dom} T:=\left\{f \in \mathcal{L}^{1}(S): T(f)<\infty\right\}$.
} 
response map $\mathcal{G}_{\eta}: \mathcal{P}(S) \rightarrow \mathcal{P}(S)$ as

$$
\mathcal{G}_{\eta}(\mathbb{P})=\left[\arg \max _{\mathbb{Q} \in \mathcal{P}(S)}\left(\mathbb{E}^{\mathbf{Q}}[\pi(\mathbb{P})]-\eta v(\mathbb{Q})\right)\right]
$$

It follows from Charalambos and Aliprantis (2013) that the extended functional $\mathbb{P} \mapsto\left(\mathbb{E}^{\mathrm{Q}}[\pi(\mathbb{P})]-\right.$ $\eta v(\mathbb{Q}))$ has a unique maximizer. Therefore, $\mathcal{G}_{\eta}$ is well-defined. Furthermore, due to the restriction that $v(\mathbb{Q})=\infty$ if $\mathbb{Q} \notin \mathcal{P}_{0}(S)$, it must be that $\mathcal{G}_{\eta}(\mathbb{P}) \in \mathcal{P}_{0}(S)$ for all $\mathbb{P} \in \mathcal{P}(S)$.

We refer to $\mathcal{G}_{\eta}(\mathbb{P})$ as the generalized perturbed best response (GPBR) to population state $\mathbb{P}$ with respect to the perturbation function $v$. Intuitively, it is a perturbation of the more usual game theoretic notion of the best response, with the extent of perturbation declining as the perturbation factor $\eta \rightarrow 0$. But it is analytically more tractable than the best response because for every $\mathbb{P} \in \mathcal{P}(S)$, it is uniquely defined. The best response, on the other hand, may not even exist at certain state in games with continuum strategy sets or, even if it exists, may be multi-valued. Using the generalized perturbed best response, we can define the generalized perturbed best response (GPBR) dynamic as

$$
\dot{\mathbb{P}}(A)=\mathcal{G}_{\eta}(\mathbb{P})(A)-\mathbb{P}(A)
$$

for $A \in \mathcal{B}(S)$. Thus, under this dynamic, a population state $\mathbb{P}$ moves in the direction of its generalized perturbed best response $\mathcal{G}_{\eta}(\mathbb{P})$. Since both $\mathcal{G}_{\eta}(\mathbb{P}), \mathbb{P} \in \mathcal{P}(S)$, it must be that $\dot{\mathbb{P}} \in$ $\mathcal{M}_{0}(S)$.

The reason behind calling $\mathcal{G}_{\eta}(\mathbb{P})$ as the generalized perturbed best response is that its construction arises as a generalization of the logit best response for games with continuous strategy sets (Mattsson and Weibull (2002)). They consider the perturbation function

$$
v(\mathbb{Q})= \begin{cases}\int_{S} q(x) \log q(x) d x & \text { if } \mathbb{Q} \in \mathcal{P}_{0}(S) \\ \infty & \text { otherwise }\end{cases}
$$

which is, of course, the Shannon entropy of $\mathbb{Q} \in \mathcal{P}(S)$. Maximizing the resulting perturbed expected payoff as in (3), we obtain the logit best response (also called logit choice measure in Lahkar and Riedel (2015)) $\mathcal{G}_{\eta}=\mathcal{L}_{\eta}$ (Mattsson and Weibull (2002)) defined as

$$
\mathcal{L}_{\eta}(\mathbb{P})(A)=\frac{\int_{A} \exp \left(\eta^{-1} \pi_{y}(\mathbb{P})\right) d y}{\int_{S} \exp \left(\eta^{-1} \pi_{x}(\mathbb{P})\right) d x}
$$

for all $A \in \mathcal{B}(S)$. The logit best response is, therefore, a particular form of the generalized 
perturbed best response $\mathcal{G}_{\eta}$, which does not assume any structural form of $v$ except that $\widetilde{v}$ is lower semicontinuous and strongly convex with respect to the $\|\cdot\|_{\mathcal{L}^{1}(S)}$ norm and that $v(\mathbb{Q})=\infty$ for all $\mathbb{Q} \notin \mathcal{P}_{0}(S) .{ }^{12}$ Applying $\mathcal{L}_{\eta}(\mathbb{P})$ to the general form of the perturbed best response dynamic (4) then gives us the continuous strategy logit dynamic $\dot{\mathbb{P}}(A)=\mathcal{L}_{\eta}(\mathbb{P})(A)-\mathbb{P}(A)$ (Perkins and Leslie (2014), Lahkar and Riedel (2015)).

Recall that $\mathcal{G}_{\eta}(\mathbb{P}) \in \mathcal{P}_{0}(S)$ for every $\mathbb{P} \in \mathcal{P}(S)$ and, therefore, always has a density function. In addition, if the existing population state $\mathbb{P}$ also has a density function, then (4) implies that $\dot{\mathbb{P}} \in \mathcal{M}_{0}(S)$ also a density function. A rest point of the dynamic (4) is a state $\mathbb{P}$ such that $\dot{\mathbb{P}}(A)=0$ for all $A \in \mathcal{B}(S)$. It is evident that any such rest point satisfies $\mathcal{G}_{\eta}(\mathbb{P})=\mathbb{P}$ and is, therefore, also a fixed point of the perturbed best response map $\mathcal{G}_{\eta}$. We call such a fixed point of $\mathcal{G}_{\eta}$ or, equivalently, a rest point of (4) a perturbed equilibrium. In the case of the logit dynamic, such an equilibrium is called a logit equilibrium.

\section{Perturbed Best Response and Perturbed Equilibria}

We now establish certain fundamental properties of the GPBR dynamic like the existence of a perturbed equilibrium and existence of solutions to the dynamic. To do so, we first define certain topological concepts that are standard in the analysis of continuous strategy evolutionary dynamics.

Two topologies arise in the context of such dynamics. One is the strong topology induced by the variational norm on $\mathcal{M}(S)$ given by $\|\mu\|_{\mathbf{T V}}=\sup _{g}\left|\int_{S} g d \mu\right|$ where $g$ is a measurable function $g: S \rightarrow \mathbb{R}$ such that $\sup _{x \in S}|g(x)| \leq 1$. If $\mathbb{P}, \mathbb{Q}$ are two probability measures, then the distance between them under this norm is (see, e.g., Shiryaev (1995))

$$
\|\mathbb{P}-\mathbb{Q}\|_{\mathbf{T V}}:=2 \sup _{A \in \mathcal{B}(S)}|\mathbb{P}(A)-\mathbb{Q}(A)|
$$

where $\|\cdot\|_{\mathbf{T V}}: \mathcal{P}(S) \times \mathcal{P}(S) \rightarrow \mathbb{R}_{+}$is called the total variation distance.

The other is the weak topology, which is induced by convergence in distribution. On way to metrize the weak topology on $\mathcal{P}(S)$ is through the Prohorov metric $\rho: \mathcal{P}(S) \times \mathcal{P}(S) \rightarrow \mathbb{R}_{+}$ defined by

$$
\rho(\mathbb{P}, \mathbb{Q}):=\inf \left\{\epsilon>0: \mathbb{P}(A) \leq \mathbb{Q}\left(A^{\epsilon}\right)+\epsilon \text { and } \mathbb{Q}(A) \leq \mathbb{P}\left(A^{\epsilon}\right)+\epsilon \text { for all } A \in \mathcal{B}(S)\right\}
$$

\footnotetext{
${ }^{12}$ The counterpart of Shannon entropy also satisfies lower semicontinuity and strong convexity as we will show later.
} 
for all $\mathbb{P}, \mathbb{Q} \in \mathcal{P}(S)$ (see, e.g., Billingsley (2013)). Thus, a sequence of probability measures $\left(\mathbb{P}_{n}\right)_{n \geq 1} \subset \mathcal{P}(S)$ converges weakly to $\mathbb{P} \in \mathcal{P}(S)$ if $\rho\left(\mathbb{P}_{n}, \mathbb{P}\right) \rightarrow 0$. We will be using the strong topology to establish results on convergence under perturbed best response dynamics, which would imply convergence under the weak topology. The weak topology, on the other hand, is useful for establishing results like existence of equilibrium since it renders the state space $\mathcal{P}(S)$ compact.

We begin with the existence of a perturbed equilibrium. For that we will require few preliminary notations and definitions. Let $\mathcal{C}(S)$ denote the collection of continuous functions on $S$ endowed with the sup-norm (see Footnote (6)). Recall that the perturbation function $v$ is not continuous in the strong topology. Hence, it is not Fréchet differentiable, which is the more general notion of differentiablity in Banach spaces (see Definition 3.4). Therefore we apply a weaker notion of differentiability called the Gâteaux derivative which is defined as follows.

Definition 3.1 (Gâteaux Differentiability). Let $\phi: \mathcal{M}(S) \rightarrow \mathbb{R}$. The Gateaux derivative $d \phi(\mu):$ $\mathcal{M}_{0}(S) \rightarrow \mathbb{R}$ of $\phi$ at a point $\mu \in \mathcal{M}(S)$ towards the direction $v \in \mathcal{M}_{0}(S)$ is defined as

$$
d \phi(\mu)(v):=\lim _{\epsilon \rightarrow 0} \frac{\phi(\mu+\epsilon v)-\phi(\mu)}{\epsilon}
$$

provided the limit exists.

If $\phi$ is Gâteaux differentiable at $\mu \in \mathcal{M}(S)$, we will assume that there exists a measurable map $\nabla^{\mathrm{G}} \phi(\mu)$ called Gâteaux gradient of $\phi$ at $\mu$ such that $\mathrm{d} \phi(\mu)(v)=\left\langle\nabla^{\mathrm{G}} \phi(\mu), v\right\rangle:=\int_{S} \nabla^{\mathrm{G}} \phi_{x}(\mu) v(d x)$.

Our next theorem (Theorem 3.1) requires us to show that the mapping $\mathbb{P} \mapsto \mathcal{G}_{\eta}(\mathbb{P})$ is continuous in the weak toplogy on $\mathcal{P}(S)$. For the purpose of this theorem, we introduce the notion of admissible perturbations which ensure lower semicontinuity of the perturbation function on $\mathcal{P}_{0}^{c}(S)$ with respect to the weak topology.

Definition 3.2 (Admissible Perturbation). Let $\pi: \mathcal{P}(S) \rightarrow \mathcal{L}^{\infty}(S)$ be a population game. We call a perturbation function $v$ admissible if $\operatorname{Range}(\pi) \subseteq \mathcal{C}(S)$ implies $\mathcal{G}_{\eta}(\mathbb{P}) \in \mathcal{P}_{0}^{c}(S)$ for all $\eta>0$ and all $\mathbb{P} \in \mathcal{P}_{0}^{c}(S) .^{13}$

We now show that a perturbed equilibrium always exists subject to some restrictions on the perturbation function $v$. The following theorem states the relevant result. The proof follows from the Brouwer-Schauder-Tychonoff fixed point theorem (see Charalambos and Aliprantis (2013) for details).

\footnotetext{
${ }^{13}$ The fact that Shannon entropy (see (5)) is an admissible perturbation function follows from (6) and Definition 3.2.
} 
Theorem 3.1. Suppose $\pi: \mathcal{P}(S) \rightarrow \mathcal{L}^{\infty}(S)$ is population game such that Range $(\pi) \subseteq \mathcal{C}(S)$. Suppose that the perturbation function $v$ is admissible and Gâteaux differentiable on $\mathcal{P}_{0}(S)$ such that the Gateaux gradient $\nabla^{G_{v}}(\mathbb{Q}) \in \mathcal{C}(S)$ for all $\mathbb{Q} \in \mathcal{P}_{0}^{c}(S)$. Then, for every $\eta>0$, the GPBR map $\mathcal{G}_{\eta}: \mathcal{P}(S) \rightarrow \mathcal{P}(S)$ admits a fixed point, i.e. there exists $\mathbb{P}_{\eta}^{\circ} \in \mathcal{P}(S)$ such that $\mathcal{G}_{\eta}\left(\mathbb{P}_{\eta}^{\circ}\right)=\mathbb{P}_{\eta}^{\circ}$. Any such $\mathbb{P}_{\eta}^{\circ}$ is a perturbed equilibrium of $\pi$.

The proof of Theorem 3.1 is in Appendix A.1. The proof requires us to show that the mapping $\mathcal{G}_{\eta}: \mathcal{P}(S) \rightarrow \mathcal{P}(S)$ is continuous in the weak topology (Lemma A.1). We show this in two steps. Firstly, under the given conditions of Theorem 3.1, we show that the perturbation function is weakly lower semicontinuous ${ }^{14}$ on $\mathcal{P}_{0}^{c}(S)$, or, equivalently lower semicontinuous on $\mathcal{P}_{0}^{c}(S)$ in the weak topology. This in turn calls for the use of the Argmax Continuous Mapping Theorem (Van Der Vaart and Wellner (1996), Ferger (2004)). It states that if one has a sequence of stochastic processes indexed by some metric space and if the sample paths of these processes are almost surely upper semicontinuous with unique maximizers, then the sequence of maximizers of these processes converges in distribution to the unique maximizer of the limiting process. Once we establish this lemma, the result follows from the Brouwer-Schauder-Tychonoff fixed point theorem and the compactness of $\mathcal{P}(S)$. Lahkar and Riedel (2015) establish a similar result on the existence of logit equilibria, which are fixed points of the logit best response. The proof of Theorem 3.1 is, however, much more general as it does not assume any functional form of the perturbation function $v$. The only requirements that Lemma A.1 imposes upon $v$ are strong convexity and lower semicontinuity.

We now consider the relationship between a perturbed equilibrium of $\pi$ and a Nash equilibrium of the game. Recall from (3) that the extent of perturbation in payoffs while calculating the perturbed best response is determined by the parameter $\eta$. It, therefore, seems plausible that lower is $\eta$, the closer is a perturbed best response to a best response and, therefore, closer is a perturbed equilibrium to a Nash equilibrium. Our next theorem verifies this intuition subject to certain conditions. We need to establish certain preliminaries before stating the theorem.

Let $\operatorname{GE}(\pi, \eta)$ be the set of perturbed equilibria of $\pi$ with respect to the GPBR map $\mathcal{G}_{\eta}: \mathcal{P}(S) \rightarrow$ $\mathcal{P}(S)$. That is, $\operatorname{GE}(\pi, \eta):=\left\{\mathbb{P}_{\eta}^{\circ} \in \mathcal{P}(S): \mathcal{G}_{\eta}\left(\mathbb{P}_{\eta}^{\circ}\right)=\mathbb{P}_{\eta}^{\circ}\right\}$. We will require the notion of the convex conjugate of $\widetilde{v}$. The general definition of the convex conjugate of a function is as follows.

Definition 3.3 (Convex conjugate of a function). Let $X$ be a real topological vector space and let $X^{*}$ be the dual space of $X$. For a function $f: X \rightarrow \mathbb{R} \cup\{\infty,-\infty\}$, the convex conjugate $f^{*}: X^{*} \rightarrow$

\footnotetext{
${ }^{14}$ A mapping $h:(\mathcal{P}(S), \rho) \rightarrow \mathbb{R}$ is weakly lower semicontinuous if for all sequences $\left(\mathbb{P}_{n}\right)_{n \geq 1}$ in $\mathcal{P}(S), \rho\left(\mathbb{P}_{n}, \mathbb{P}\right) \rightarrow 0$

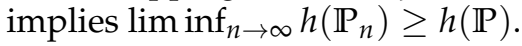


$\mathbb{R} \cup\{\infty,-\infty\}$ is defined by

$$
f^{*}\left(x^{*}\right):=\sup _{x \in X}\left(x^{*}(x)-f(x)\right)
$$

Following Definition 3.3, we denote the convex conjugate of $\widetilde{v}$ as $\widetilde{v}^{*}$. We will also use the notation $v_{\eta}$ to denote $\eta v .{ }^{15}$ In that case, $\widetilde{v}_{\eta}^{*}$ is the convex conjugate of the perturbation counterpart $\widetilde{v}_{\eta}$.

We will also require the notion of the Fréchet derivative, which is a generalization of the usual derivative to Banach spaces. The Fréchet derivative is defined as follows.

Definition 3.4 (Fréchet Differentiablity). Let $V$ and $W$ be normed linear spaces. An operator $\phi: V \rightarrow$ $W$ is Fréchet differentiable at $x$ if there exists a bounded linear operator $A: V \rightarrow W$ such that

$$
\lim _{\|h\|_{V} \rightarrow 0} \frac{\|\phi(x+h)-\phi(x)-A h\|_{W}}{\|h\|_{V}}=0 .
$$

Thus, if $A$ is the Fréchet derivative of $\phi$, then $\phi(x+h)=\phi(x)+A(h)+\mathbf{o}\left(\|h\|_{V}\right)$ for all $h$ near zero in $V$. In this paper, we consider Fréchet differentiability of functions $\phi: \mathcal{M}(S) \rightarrow \mathbb{R}$. We denote the Fréchet derivative of such a function by $\mathrm{D} \phi$.

If $\phi$ is Fréchet differentiable, we will assume that there exists a bounded measurable map $\nabla^{\mathbf{F}} \phi(\mu) \in \mathcal{L}^{\infty}(S)$ such that for all $v \in \mathcal{M}_{0}(S)$, we have

$$
\mathrm{D} \phi(\mu) v:=\int_{S} \nabla^{\mathbf{F}} \phi(\mu) d v=\int_{S} \nabla^{\mathbf{F}} \phi(\mu)(x) v(d x)=\left\langle\nabla^{\mathbf{F}} \phi(\mu), v\right\rangle .
$$

We then call the map $\nabla^{\mathbf{F}} \phi$ the Fréchet gradient of $\phi$.

With these notations, we now define the following two important conditions, Condition A1 and Condition A2, that will be required for establishing convergence of perturbed equilibria to Nash equilibrium. Intuitively, these conditions impose some restrictions on the perturbation function so that small changes in the population state does not lead to large changes in the perturbation. Recall that $\widetilde{v}$ (Definition 2 ) denotes the perturbation counterpart of $v$, and $\widetilde{v}^{*}$ denotes the convex conjugate of $\widetilde{v}$ (Definition 3.3).

Definition 3.5 (Condition A1). A perturbation function $v$ satisfies Condition A1 if for all $\eta>0$, all disjoint $E, F \in \mathcal{B}(S)$ and all $g \in \mathcal{L}^{\infty}(S)$ with $\inf _{s \in F} g(s)>\sup _{t \in E} g(t)$, there exists a function $u:(0, \infty) \rightarrow \mathbb{R}$ with $\lim _{x \rightarrow 0} u(x)=\infty$ such that

$$
D \widetilde{v}_{\eta}^{*}(g)\left(\mathbb{I}_{F}\right) \geq u(\eta) D \widetilde{v}_{\eta}^{*}(g)\left(\mathbb{I}_{E}\right),
$$

\footnotetext{
${ }^{15}$ Notice from, for example, (5) that the original perturbation function $v$ is defined independent of $\eta$. This additional piece of notation is required to capture the effects as $\eta \rightarrow 0$.
} 
where $D \widetilde{v}_{\eta}^{*}(g)$ is the Fréchet derivative of $\widetilde{v}_{\eta}^{*}(g)$, and $\mathbb{I}_{E}, \mathbb{I}_{F}$ are indicator functions on $E$ and $F$ respectively. Before defining Condition A2, we required another definition; that of the shift of a probability measure. It is defined as follows.

Definition 3.6. Let $\mathbb{P} \in \mathcal{P}_{0}(S)$ and let $E, F \subseteq S$ be disjoint. We say that $\widetilde{\mathbb{P}}$ is a shift of $\mathbb{P}$ from $E$ to $F$ if

(i) $\widetilde{\mathbb{P}}(E)<\mathbb{P}(E)$,

(ii) $\widetilde{\mathbb{P}}(F)=\mathbb{P}(F)+(\mathbb{P}(E)-\widetilde{\mathbb{P}}(E))$, and

(iii) $\widetilde{\mathbb{P}}(G)=\mathbb{P}(G)$ for all $G \subseteq S \backslash(E \cup F)$.

Condition A2 is now defined as follows.

Definition 3.7 (Condition A2). We say that a perturbation function satisfies Condition A2 with respect to a sequence of probability measures $\left(\mathbb{P}_{n}\right)_{n \geq 1}$ if for all disjoint $E, F \subseteq S$ with $\lim _{n \rightarrow \infty} \mathbb{P}_{n}(E) \neq 0$ and $\inf _{z \in F} \pi_{z}\left(\mathbb{P}_{n}\right)>\sup _{w \in E} \pi_{w}\left(\mathbb{P}_{n}\right)$ for sufficiently large $n$, there exist $\left(A_{n}\right)_{n \geq 1} \subseteq E$ and $\left(\widetilde{\mathbb{P}}_{n}\right)_{n \geq 1}$ where $\widetilde{\mathbb{P}}_{n}$ is a shift of $\mathbb{P}_{n}$ from $A_{n}$ to $F$, and a constant $\kappa>0$ such that for sufficiently large $n$,

$$
\left|v\left(\mathbb{P}_{n}\right)-v\left(\widetilde{\mathbb{P}}_{n}\right)\right| \leq \kappa\left(\mathbb{P}_{n}\left(A_{n}\right)-\widetilde{\mathbb{P}}_{n}\left(A_{n}\right)\right)
$$

With these preliminaries, we now state our desired theorem on convergence of perturbed equilibria to a Nash equilibria.

Theorem 3.2. Suppose that the conditions of Theorem 3.1 are satisfied. Let $\left(\eta_{n}\right)_{n \geq 1}$ be any positive sequence such that $\eta_{n} \downarrow 0$ and let $\left(\mathbb{P}_{n}^{\circ}\right)_{n \geq 1}$ denote the corresponding sequence of perturbed equilibria. Suppose also that the perturbation function $v$ satisfies Condition A1, or Condition A2 with respect to $\left(\mathbb{P}_{n}^{\circ}\right)_{n \geq 1}$, as stated in Definitions 3.5 and 3.7 respectively. Then the family $G E\left(\pi, \eta_{n}\right)_{n \geq 1}$ of perturbed equilibria has accumulation points under the weak topology and any such accumulation point is a Nash equilibrium of the original game.

The proof of this theorem is in Appendix A.2. We provide some intuition as to how Condition A1 and Condition A2 are relevant for the proof. Since the space of signed measures is infinite dimensional, assuming lower semicontinuity and strong convexity under the strong topology does not guarantee continuity of the perturbation function. However, some type of continuity of the perturbation function is required to ensure the convergence of the perturbed equilibria to the Nash equilibrium of the underlying game. To ensure such weaker form of continuity of the perturbation function, we impose two conditions, namely A1 and A2. The weaker form of 
continuity we consider implies that when a small proportion of agents switch their strategies from one set to another, the change in the perturbation function will also be small. This guarantees that the change in the perturbation function caused by small shift of the agents' strategies will be under control, and as a consequence, the payoff from the shifted strategies can be made to dominate that change. Therefore, when perturbation decreases gradually, agents switch to exercising strategies in those subsets which have a greater payoff and thereby will weakly converge to a Nash equilibrium of the underlying game.

\subsection{EXAMPLES OF PERTURbATION FunCtions}

We now provide some examples of perturbation functions $v: \mathcal{P}(S) \rightarrow \mathbb{R} \cup\{\infty\}$ that are admissible and that satisfy Conditions A1 or A2. Theorem 3.2 would then be valid for with respect to these perturbation functions.

Example 3.1 (Shannon entropy). The first example is the Shannon entropy itself as defined in (5) and upon which the canonical logit dynamic is based.

We now establish that Shannon entropy satisfies Condition $A 1$ (Definition 3.5). Let $g \in \mathcal{L}^{\infty}(S)$ and let $E, F \in \mathcal{B}(S)$ be disjoint such that $\inf _{s \in F} g(s)>\sup _{t \in E} g(t)$. Then

$$
\frac{D \widetilde{v}_{\eta}^{*}(g)\left(\mathbb{I}_{F}\right)}{D \widetilde{v}_{\eta}^{*}(g)\left(\mathbb{I}_{E}\right)}=\frac{\int_{F} \exp \left(\eta^{-1} g(x)\right) d x}{\int_{E} \exp \left(\eta^{-1} g(y)\right) d y} \geq \exp \left(\left(\inf _{s \in F} g(s)-\sup _{t \in E} g(t)\right) / \eta\right) \frac{\lambda(F)}{\lambda(E)}
$$

where $\lambda$ denotes the Lebesgue measure. Therefore Shannon entropy satisfies Condition $(A 1)$ with $u(\eta)=$ $\exp \left(\left(\inf _{s \in F} g(s)-\sup _{t \in E} g(t)\right) / \eta\right) \frac{\lambda(F)}{\lambda(E)}$ for all $\eta>0$.

We know from Lahkar and Riedel (2015) that under the Shannon entropy, a sequence of the relevant perturbed equilibria, which is the logit equilibria, converges to Nash equilibria as $\eta \rightarrow 0$. The exercise in Example 3.1 provides another demonstration, albeit indirect, of this fact. Such convergence is a consequence of the more general Condition A1. We now present two additional entropy functions which, as far as we know, has not been explored much in the literature on evolutionary game theory. These are the Tsallis $(\gamma)$ entropy and the Burg entropy. In Appendix A.3, we show that these two entropy functions satisfy Condition A2 (Definition 3.7).

Example 3.2 (Tsallis $(\gamma)$ entropy). For $0<\gamma<1$, the Tsallis $(\gamma)$ entropy is defined as

$$
v^{t}(\mathbb{Q})= \begin{cases}\int_{S} \frac{q(x)-q(x)^{\gamma}}{\gamma(1-\gamma)} d x & \text { if } \mathbb{Q} \in \mathcal{P}_{0}(S) \\ \infty & \text { otherwise. }\end{cases}
$$


We now show that Tsallis $(\gamma)$ entropy is an admissible perturbation function. It follows from Héliou et al. (2020) that under perturbation by Tsallis $(\gamma)$ entropy,

$$
\mathcal{G}_{\eta}(\mathbb{P})(A)=\left[\frac{\eta}{\gamma(1-\gamma)}\right]^{1 /(1-\gamma)} \int_{A} \frac{1}{\left(\theta_{\eta}-\pi_{z}(\mathbb{P})\right)^{1 /(1-\gamma)}} d z
$$

for all $A \in \mathcal{B}(S)$, where $\theta_{\eta}$ is such that the RHS in the above equation is a valid probability density function on $S$. It follows from the above expression that Tsallis $(\gamma)$ entropy is admissible.

It is well known in the literature (see Tsallis (1988)) that the Shannon entropy (Example 3.1) can be obtained from the Tsallis $(\gamma)$ entropy (Example 3.2) by taking $\gamma \rightarrow 1$. However, the analytical properties of these entropies are quite different. Tsallis entropy is used in Héliou et al. (2020) in the context of online non-convex optimization problems.

Example 3.3 (Burg entropy). The Burg entropy is defined as

$$
v^{b}(\mathbb{Q})= \begin{cases}-\int_{S} \log q(x) d x & \text { if } \mathbb{Q} \in \mathcal{P}_{0}(S) \\ \infty & \text { otherwise }\end{cases}
$$

The fact that Burg entropy is admissible follows from Héliou et al. (2020) by using arguments similar to the Tsallis entropy.

The definition of Burg entropy for games with continuum strategy spaces (Example 3.3) is motivated from the definition of Burg entropy for games on finite strategy spaces. Suppose that the strategy space $S=\{1, \ldots, n\}$ is finite and let $\Delta:=\left\{\mathbf{x}=\left(x_{1}, \ldots, x_{n}\right) \in[0,1]^{n} \mid x_{1}+\ldots+x_{n}=\right.$ $1\}$ be the collection of population states. In such a scenario, the Burg entropy is defined as $v^{\mathbf{b}}(\mathbf{x}):=-\sum_{i=1}^{n} \log x_{i}$, for all $\mathbf{x} \in \Delta$. Hofbauer and Sandholm (2002) consider such an entropy to derive a form of finite strategy perturbed best response dynamic through stochastic perturbation. In contrast, we apply the Burg entropy as a deterministic perturbation.

It is well-known that all the three entropies mentioned in this section (namely, Shannon, Tsallis, and Burg) satisfy strong convexity and lower semicontinuity (see Héliou et al. (2020) for details). Moreover, the fact that the Tsallis and Burg entropies satisfy Condition A2, as shown in Appendix A.3, imply that it is not just the Shannon entropy to which Theorem 3.2 applies. Instead, we can meaningfully define perturbed best responses for other entropies as well and approximate Nash equilibria with their fixed points. This makes our exercise of generalizing perturbed best response dynamics beyond the logit dynamic a non-vacuous one. 


\section{Fundamental Properties of Perturbed Best Response Dynamics}

We now seek to establish the fundamental properties of the GPBR dynamic (4). These are (i) the existence of a unique solution trajectory from every initial state in $\mathcal{P}(S)$ and (ii) the continuity of such solution trajectories with respect to initial state. We will require the following definitions for establishing the relevant result.

Definition 4.1 (Lipschitz population game). A population game $\pi: \mathcal{P}(S) \rightarrow \mathcal{L}^{\infty}(S)$ is $\beta$-Lipschitz continuous if there exists a real number $\beta>0$ such that

$$
\|\pi(\mathbb{P})-\pi(\mathbb{Q})\|_{\infty} \leq \beta\|\mathbb{P}-\mathbb{Q}\|_{T V}
$$

for all $\mathbb{P}, \mathbb{Q} \in \mathcal{P}(S)$, where $\|\cdot\|_{T V}$ is the total variation distance as defined in (7).

Definition 4.2 (Semiflow of a dynamic). Consider an abstract differential equation

$$
\Gamma^{\prime}(t)=\mathcal{H}(\Gamma(t))
$$

on a Banach space $\left(X,\|\cdot\|_{X}\right)$. Suppose that a unique solution to the above differential equation exists for every initial condition $\Gamma(0)=\gamma($ say). Denote the solution of the above differential equation with initial condition $\gamma$ by $\Gamma_{\gamma}(t)$. Then the semiflow of the dynamic is the map $\zeta: X \times[0, \infty) \rightarrow X$ defined by $\zeta(\gamma, t)=\Gamma_{\gamma}(t)$ for all $\gamma \in X$ and $t \in[0, \infty)$.

We will apply Definitions 4.1 and 4.2 in establishing the fundamental properties of the GPBR dynamic. Before that, we note from (4) that if indeed the GPBR dynamic admits a solution, then $\mathcal{P}(S)$ must be forward invariant under that dynamic. This means from any initial state in $\mathcal{P}(S)$, the solution trajectory must lie in $\mathcal{P}(S)$ for all times $t \geq 0$. This is immediate because from the definition of the perturbed best response (3), if $\mathbb{P} \in \mathcal{P}(S)$, then $\mathcal{G}_{\eta}(\mathbb{P}) \in \mathcal{P}(S)$.

While forward invariance of $\mathcal{P}(S)$ is obvious, we can actually establish a stronger result which will also be useful for later in establishing the fundamental properties of the dynamic. For this purpose, recall that $\mathcal{P}_{0}(S) \subset \mathcal{P}(S)$ is the set of absolutely continuous probability measures. Let $D>1$. We then define the set

$\mathcal{P}_{D}(S):=\left\{\mathbb{P} \in \mathcal{P}_{0}(S): \frac{1}{D} \leq p(x) \leq D\right.$ for all $x \in S$ and $|p(x)-p(y)| \leq D|x-y|$ for all $\left.x, y \in S\right\}$.

Thus, $\mathcal{P}_{D}(S)$ is the set of absolutely continuous probability measures with $D$-Lipschitz continuous 
density functions and whose lower and upper bounds are given by $\frac{1}{D}$ and $D$ respectively. It is known (see Perkins and Leslie (2014), Hanche-Olsen and Holden (2010)) that $\mathcal{P}_{D}(S)$ is a compact subset of $\mathcal{P}(S)$ under the total variation norm (7).

We now show that the GPBR dynamic is forward invariant not just in $\mathcal{P}(S)$ but also $\mathcal{P}_{D}(S)$. Thus, if $\mathbb{P} \in \mathcal{P}_{D}(S)$, then the GPBR $\mathcal{G}_{\eta}(\mathbb{P}) \in \mathcal{P}_{D}(S)$ as well. ${ }^{16}$ Thus, if the GPBR dynamic admits a solution, then the entire trajectory originating in $\mathcal{P}_{D}(S)$ must lie in $\mathcal{P}_{D}(S)$ at all time $t \geq 0$. To arrive at this result, we will require a further piece of notation. Recall the set $\mathcal{L}_{+}^{1,1}(S) \subset \mathcal{L}^{1}(S)$ from (1). We now define the following subset of $\mathcal{L}_{+}^{1,1}(S)$ by imposing bounds $\frac{1}{D}$ and $D$ on the functions in $\mathcal{L}_{+}^{1,1}(S)$ and which are $D$-Lispchitz continuous, where $D>1$. Thus,

$\mathcal{L}_{+, D}^{1,1}(S):=\left\{f \in \mathcal{L}_{+}^{1,1}(S): \frac{1}{D} \leq f(x) \leq D\right.$ for all $x \in S$ and $|f(x)-f(y)| \leq D|x-y|$ for all $\left.x, y \in S\right\}$.

It is easy to note that $\xi$ restricted to $\mathcal{L}_{+, D}^{1,1}(S)$ is a bijection onto $\mathcal{P}_{D}(S)$. The following lemma states the result on forward invariance. Recall that $\widetilde{v}$ is the counterpart of $v$ (see 2 ), $\widetilde{v}^{*}$ is the convex conjugate of $\widetilde{v}$ (see Definition 3.3) and $\nabla^{\mathbf{F}} \widetilde{v}^{*}$ is the gradient. The proof is in Appendix A.4.1.

Lemma 4.1. Let $\pi: \mathcal{P}(S) \rightarrow \mathcal{L}^{\infty}(S)$ and suppose that $\nabla^{F_{v^{*}}}(\mathbb{P}) \in \mathcal{L}_{+, D}^{1,1}(S)$ for all $\mathbb{P} \in \mathcal{P}_{D}(S)$. Consider the GPBR dynamic (4). If $\mathbb{P} \in \mathcal{P}_{D}(S)$, then $\mathcal{G}_{\eta}(\mathbb{P}) \in \mathcal{P}_{D}(S)$.

Thus, Lemma 4.1 would imply that if indeed the generalized PBR dynamic admits a solution, then $\mathcal{P}_{D}(S)$ would be invariant. The following theorem shows that indeed such solutions exist. Moreover, any such solution must be unique and continuous with respect to initial states. This is the main result of this section. The proof, which follows from the Picard-Lindelöf theorem, is in Appendix A.4.2.

Theorem 4.1. Suppose that $\pi: \mathcal{P}(S) \rightarrow \mathcal{L}^{\infty}(S)$ is a $\beta$-Lipschitz population game and $v$ is a deterministic perturbation function. Suppose also that the counter part $\widetilde{v}$ of $v$ is closed ${ }^{17}$ and L-strongly convex (see footnote 11). Then, given any initial condition $\mathbb{P}_{0} \in \mathcal{P}(S)$, there exists a unique solution to the GPBR dynamic $\dot{\mathbb{P}}=\mathcal{G}_{\eta}(\mathbb{P})-\mathbb{P}$. Furthermore, the semiflow of the dynamic is continuous in the initial condition $\mathbb{P}_{0}$ with respect to the total variation norm.

\footnotetext{
${ }^{16}$ Perkins and Leslie (2014) establish this result for the specific case of the logit best response. Our result is more general.

${ }^{17}$ We call a function $f:(X, \tau) \rightarrow(\mathbb{R},|\cdot|)$ closed if the set $\{(x, t): x \in X, t \in \mathbb{R}, t \geq f(x)\}$ is a closed subset of $X \times \mathbb{R}$.
} 


\section{Convergence in Potential Games}

We now establish convergence of the GPBR dynamic to perturbed equilibria in potential games (Monderer and Shapley (1996), Cheung (2014), Lahkar and Riedel (2015)). In order to define such games, recall from (9) our assumption that a Fréchet differentiable function $\phi$ has a Fréchet gradient $\nabla^{\mathrm{F}} \phi$.

Definition 5.1 (Potential Games). A population game $\pi: \mathcal{P}(S) \rightarrow \mathcal{L}^{\infty}(S)$ is said to be a potential game if there exists a Fréchet Differentiable functional $\varphi: \mathcal{M}(S) \rightarrow \mathbb{R}$ such that

$$
\nabla^{F} \varphi(\mathbb{P})=\pi(\mathbb{P}) \quad \text { for all } \mathbb{P} \in \mathcal{P}(S) .
$$

The functional $\varphi$ is called the potential function of the game $\pi$.

Definition 3.4 would then imply that

$$
\mathrm{D} \varphi(\mathbb{P})(v):=\int_{S} \nabla^{\mathbf{F}} \varphi(\mathbb{P}) d v=\int_{S} \nabla^{\mathbf{F}} \varphi(\mathbb{P})(x) v(d x)=\int_{S} \pi_{x}(\mathbb{P}) v(d x)=:\langle\pi(\mathbb{P}), v\rangle .
$$

In order to establish the desired convergence result, we introduce the entropy adjusted potential function defined as follows.

Definition 5.2 (Entropy adjusted potential function). For a potential game $\pi: \mathcal{P}(S) \rightarrow \mathcal{L}^{\infty}(S)$, we define the entropy adjusted potential function $\widetilde{\varphi}: \mathcal{P}_{0}(S) \rightarrow \mathbb{R}$ as $\widetilde{\varphi}(\mathbb{P}):=\varphi(\mathbb{P})-v_{\eta}(\mathbb{P})$. For some $D>1$, the restriction of $\widetilde{\varphi}$ to $\mathcal{P}_{D}(S)$ is denoted by $\widetilde{\varphi}_{D}$.

Recall that since the perturbation function $v$ is not Fréchet differentiable, we defined the Gateaux derivative (Definition 3.1) which is a generalization of the directional derivative and which will allow us to consider movements along only specific directions; for example, directions that have density functions that are bounded and bounded away from zero (as in $\mathcal{P}_{D}(S)$ ). Therefore, we apply the Gateaux derivative to analyze the entropy adjusted potential function.

We need an additional result for establishing convergence in potential games. We say that the GPBR dynamic satisfies positive correlation with respect to a function $\Gamma: \mathcal{P}(S) \rightarrow \mathcal{L}^{\infty}(S)$ if $\int_{S} \Gamma_{x}(\mathbb{P}) \dot{\mathbb{P}}(d x) \geq 0$ for all $\mathbb{P} \in \mathcal{P}(S)$ with equality only if $\dot{\mathbb{P}}=0$. Define the virtual payoff of strategy $x$ at state $\mathbb{P}$ as

$$
\tilde{\pi}_{x}(\mathbb{P})=\pi_{x}(\mathbb{P})-\nabla^{\mathbf{G}_{v_{\eta}}}(\mathbb{P})(x) .
$$

The following lemma shows that the dynamic satisfies positive correlation with respect to the virtual payoff. In order to state the lemma, we impose a further condition, stated in (13), 
on our perturbation functions. Let $\mathcal{C}^{1}([0, \infty))$ be the collection of all continuously differentiable functions on $[0, \infty)$. Suppose that there exists a $\mathcal{C}^{1}([0, \infty))$ function $\Theta:[0, \infty) \rightarrow \mathbb{R}$ such that the perturbation function $v$ can be expressed as

$$
v(\mathbb{P})= \begin{cases}\int_{S} \Theta \circ p(x) d x & \text { if } \mathbb{P} \in \mathcal{P}_{0}(S) \\ \infty & \text { otherwise. }\end{cases}
$$

The three entropies we consider, namely the Shannon entropy, the Tsallis entropy and the Burg entropy, do satisfy (13). We then have the following lemma. The proof is in Appendix A.5.

Lemma 5.1. Let $\pi: \mathcal{P}(S) \rightarrow \mathcal{L}^{\infty}(S)$ be a potential game. Suppose that the perturbation function $v$ satisfies (13). Then the followings hold:

(i) Suppose that $\Theta$ is either strictly increasing or strictly decreasing on $[0, \infty)$. Then for every $\eta>0$, the generalized dynamic satisfies positive correlation with respect to the virtual payoff $\tilde{\pi}$ as defined in (12). Thus, $\int_{S} \widetilde{\pi}_{x}(\mathbb{P}) \dot{\mathbb{P}}(d x) \geq 0$ for all $\mathbb{P} \in \mathcal{P}(S)$ with equality only if $\dot{\mathbb{P}}=0$.

(ii) Suppose that there exists $0 \leq \alpha<1<\beta<\infty$ such that $\Theta$ is either strictly increasing or strictly decreasing on $[\alpha, \beta)$. Then for every $\eta>0$ such that $\left[D_{\eta}^{-1}, D_{\eta}\right] \subset[\alpha, \beta)$, the generalized dynamic satisfies positive correlation with respect to the virtual payoff $\tilde{\pi}$ as defined in (12). Thus, $\int_{S} \widetilde{\pi}_{x}(\mathbb{P}) \dot{\mathbb{P}}(d x) \geq 0$ for all $\mathbb{P} \in \mathcal{P}(S)$ with equality only if $\dot{\mathbb{P}}=0$.

Intuitively, this implies that there is a positive relationship between the virtual payoff of a strategy and it rate of change under the generalized PBR dynamic. It is a generalization of the corresponding result for the logit dynamic in Lahkar and Riedel (2015) to more generalized PBR dynamics. Lemma 5.1 lemma leads to the following theorem on convergence in potential games. The proof is in Appendix A.5.

Theorem 5.1. Let $\pi: \mathcal{P}(S) \rightarrow \mathcal{L}^{\infty}(S)$ be a potential game with potential function $\varphi$ which is Frechet differentiable. Let $v$ be a deterministic perturbation function such that $\widetilde{v}$ is closed. Suppose further that the Frechet derivative of the convex conjugate $\widetilde{v}^{*}$ of $\widetilde{v}$ is identified by some element in $\mathcal{L}_{+, D}^{1,1}(S)$ for some $D>1$. Let (13) be satisfied so that Lemma 5.1 holds. Then the following hold.

(i) $\widetilde{\varphi}_{D}$ increases weakly along every solution trajectory of the GPBR dynamic (4) arising in $\mathcal{P}_{D}(S)$ and increases strictly across every non stationary solution trajectory.

(ii) The set of $\omega$-limit points (in the strong topology on $\mathcal{P}_{D}(S)$ ) of any trajectory of the GBPR dynamic is a non-empty connected compact set of perturbed equilibria. Moreover, such limit points are local maximizers of the entropy-adjusted potential function in $\mathcal{P}_{D}(S)$. 


\section{Convergence in Negative Semidefinite Games}

In this section, we establish convergence of the GPBR dynamic to perturbed equilibria in negative semidefinite games. We first recall the definition of such games from Lahkar and Riedel (2015).

Definition 6.1. A population game $\pi: \mathcal{P}(S) \rightarrow \mathcal{L}^{\infty}(S)$ is said to be negative semidefinite if

$$
\int_{S}\left(\pi_{x}(\mathbb{Q})-\pi_{x}(\mathbb{P})\right)(\mathbb{Q}-\mathbb{P})(d x) \leq 0
$$

for all $\mathbb{P}, \mathbb{Q} \in \mathcal{P}(S)$.

If the inequality in (14) holds strictly, then we call the game negative definite. Two examples of negative semidefinite games are linear quadratic symmetric normal form games with payoff function $\varphi(x, y)=-x^{2}+a x y$, for some constant $a \leq 0$ (Hofbauer et al. (2009)) and random matching in a two-player symmetric zero-sum game (Cheung (2014)).

Suppose that the population game $\pi: \mathcal{P}(S) \rightarrow \mathcal{L}^{\infty}(S)$ is $C^{1}$ in the sense of Fréchet differentiability with respect to the appropriate topology under consideration. Then it is well known (see Cheung (2014), Lahkar and Riedel (2015)) that $\pi$ is negative semidefinite if and only if

$$
\langle\mathrm{D} \pi(\mathbb{P}) \mu, \mu\rangle \leq 0 \text { for all } \mathbb{P} \in \mathcal{P}(S) \text { and } \mu \in \mathcal{M}_{0}(S) .
$$

The following lemma shows that such games have a unique perturbed equilibrium. The proof, in Appendix A.6, is a generalization of a similar result in Lahkar and Riedel (2015) for the logit equilibrium. That result in Lahkar and Riedel (2015) is itself an extension of the result about uniqueness of perturbed equilibrium in finite strategy negative semidefinite games as established in Hofbauer and Sandholm (2007).

Lemma 6.1. Let $\eta>0$ and $D>1$. Let $\pi: \mathcal{P}(S) \rightarrow \mathcal{L}^{\infty}(S)$ be a negative semidefinite game which $C^{1}$ in the sense of Frechet differentiability. ${ }^{18}$ Suppose that the perturbation function $v$ be Gâteaux differentiable such that for every $\epsilon>0,\left\langle d \nabla^{G} v_{\eta}(\mathbb{P}+\epsilon \mu), \mu\right\rangle \geq 0$ for all $\mathbb{P} \in \mathcal{P}_{0}(S)$ and all absolutely continuous $\mu \in \mathcal{M}_{0}(S)$. Then the game $\pi$ has a unique perturbed equilibrium.

The three entropies considered in this paper, Shannon, Tsallis, and Burg, satisfy the conditions

\footnotetext{
${ }^{18}$ For the purpose of this lemma, we extend the domain of the definition of $\pi$ to $\mathcal{M}(S)$.
} 
in Lemma 6.1. ${ }^{19}$ Therefore, in each of these cases, the associated perturbed best response leads to a unique perturbed equilibrium in negative semidefinite games.

It is known that a negative semidefinite game has a convex set of Nash equilibria (Hofbauer and Sandholm (2009)). In fact, if the game is negative definite, then there exists a unique Nash equilibrium. Lemma 6.1, however, shows that when it comes to perturbed equilibria, the game does not have to be negative definite for the equilibrium to be unique. Even if (14) is satisfied weakly so that the game is only negative semidefinite, we still have uniqueness of perturbed equilibrium.

We now establish convergence of the generalized perturbed best response dynamic to the unique perturbed equilibrium, as shown in Lemma 6.1, in negative semidefinite games. For this purpose, we adopt a similar approach as in the case of potential games and prescribe a Lyapunov function which satisfy certain monotonicity properties. Following Lahkar and Riedel (2015) we define the Lyapunov function for a negative semidefinite game $\pi$ as ${ }^{20}$

$$
\Lambda(\mathbb{P}):=\left(\int_{S} \pi_{x}(\mathbb{P}) \mathcal{G}_{\eta}(\mathbb{P})(d x)-v_{\eta}\left(\mathcal{G}_{\eta}(\mathbb{P})\right)\right)-\left(\int_{S} \pi_{x}(\mathbb{P}) \mathbb{P}(d x)-v_{\eta}(\mathbb{P})\right)
$$

Since the perturbation function $v$ is admissible (Definition 3.2), $\mathcal{G}_{\eta}(\mathbb{P}) \in \mathcal{P}_{0}(S)$ for all $\mathbb{P} \in \mathcal{P}_{0}(S)$. Also, since the perturbation function $v$ is finite on $\mathcal{P}_{0}(S)$, we have $\Lambda$ is well defined.

We show that the Lyapunov function defined in (15) decreases along every solution trajectory to the GPBR dynamic and decreases strictly along every non-stationary solution. This is stated in the following theorem, which is the main result of this section. The proof is in Appendix A.6.

Theorem 6.1. Let $\pi: \mathcal{P}(S) \rightarrow \mathcal{L}^{\infty}(S)$ be a negative semidefinite population game that is $C^{1}$ in the sense of Fréchet differentiability. Consider the GBPR dynamic (4) and suppose the perturbation function satisfies the conditions of Lemma 6.1. Let $\Lambda_{D}: \mathcal{P}_{D}(S) \rightarrow R$ be the restriction of $\Lambda$ (see (15)) to $\mathcal{P}_{D}(S)$. This function decreases monotonically to zero along every solution trajectory of the dynamic that originates in $\mathcal{P}_{D}(S)$, and decreases strictly along nonstationary solutions. Hence, every solution trajectory of the GPBR dynamic in $\mathcal{P}_{D}(S)$ converges, with respect to the strong topology on $\mathcal{P}_{D}(S)$, to the unique perturbed

${ }^{19}$ Fix $\epsilon>0$. Let $\mathbb{P} \in \mathcal{P}_{0}(S)$ and $\mu \in \mathcal{M}_{0}(S)$ with densities $p($.$) and z($.$) respectively. It is known from Lahkar and$ Riedel (2015) that for Shannon entropy, $\nabla^{\mathbf{G}_{v_{\eta}}}(\mathbb{P}+\epsilon \mu)(x)=\eta(1+\log (p(x)+\epsilon \mu(x)))$ for all $x \in S$. It then follows

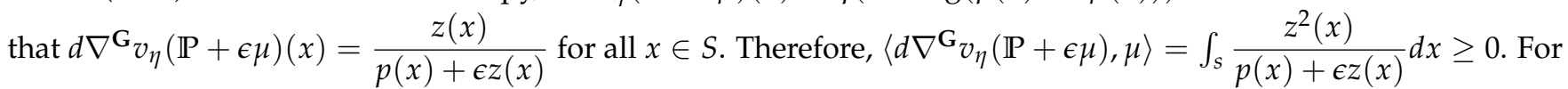
Tsallis and Burg entropy, $d \nabla^{\mathbf{G}} v_{\eta}(\mathbb{P}+\epsilon \mu)(x)$ is equal to $\frac{\eta \gamma(1-\gamma)}{(p(x)+\epsilon z(x))^{2-\gamma}}$ and $\frac{\eta}{(p(x)+\epsilon z(x))^{2}}$, respectively, for all $x \in S$. The result then follows for these two entropies through similar arguments.

${ }^{20}$ The Lyapunov function in Lahkar and Riedel (2015) is defined with respect to the logit dynamic and is an extension of the Lyapunov function in Hofbauer and Hopkins (2005) for finite strategy negative semidefinite games. The function in (15) is for the general class of perturbed best response dynamics. 
equilibrium of the game $\pi$.

Theorem 6.1 generalizes the result on convergence of the logit dynamic (Lahkar and Riedel (2015)) in negative semidefinite games to all continuous strategy perturbed best response dynamics considered in this paper. It is, therefore, the continuous strategy analog of the result obtained by Hofbauer and Sandholm (2007) for all perturbed best response dynamics in finite strategy negative semidefinite games.

\section{CONCLUSION}

This paper has considered a generalization of the logit dynamic in large population games with a continuum of strategies. We have considered general deterministic perturbations that satisfy lower semicontinuity and strong convexity. Apart from the well known Shannon entropy, other perturbations that satisfy these conditions are the Tsallis entropy and the Burg entropy. Once we perturb payoffs using such perturbation functions and allow agents to best respond, we obtain the generalized perturbed best response (GPBR) and the associated dynamic. This constitutes our generalization of the logit dynamic.

We characterize the rest points of the GPBR dynamic, which we call perturbed equilibria, and show that they approximate Nash equilibria of the underlying population game as the extent of the perturbation becomes small. Establishing this result requires us to impose appropriate lower bounds on the perturbations we consider. We then establish the fundamental properties of the GPBR dynamic; namely, existence and uniqueness of solution trajectories, and their continuity with respect to initial conditions. Finally, we establish convergence of the GPBR dynamic in potential games and negative semidefinite games.

This paper has been confined to deterministic perturbations of the best response. An interesting research question for the future is to derive perturbed best response dynamics using stochastic perturbations. This exercise has led to interesting results in finite strategy large population games (Hofbauer and Sandholm (2002)). But so far, this question has remained unexplored in games with continuous strategy sets. 


\section{A. APPENDIX}

\section{A.1 Proof of TheOrem 3.1}

The proof of Theorem 3.1 requires the application of the Brouwer-Schauder-Tychonoff fixed point theorem (see Charalambos and Aliprantis (2013) for details), which we state below.

Theorem A.1 (Brouwer-Schauder-Tychonoff FPT). Let $V$ be a non-empty compact convex subset of a locally convex Hausdorff space, and let $f: V \rightarrow V$ be a continuous function. Then the set of fixed points of $f$ is compact and nonempty.

We apply Theorem A.1 with $V=\mathcal{P}(S)$ and $f(\mathbb{P})=\mathcal{G}_{\eta}(\mathbb{P})$. Recall the Prohorov metric $\rho$. Since $(\mathcal{P}(S), \rho)$ is a metric space, it is Hausdorff. Furthermore, it is convex by definition. To show that $f$ is continuous in the weak topology, it is sufficient to show that $\mathcal{G}_{\eta}$ is continuous in the weak topology. We show this in the following lemma, which is the key result in the proof of Theorem 3.1.

Lemma A.1. Suppose that the conditions in Theorem 3.1 are satisfied. Then the mapping $\mathcal{G}_{\eta}: \mathcal{P}(S) \rightarrow$ $\mathcal{P}(S)$ is continuous in the weak topology.

The proof of Lemma A.1 will, in turn, require the following theorem.

Theorem A.2 (Argmax Continuous Mapping Theorem, Van Der Vaart and Wellner (1996), Ferger (2004)). Let $\left(\mathbb{M}_{n}\right)_{n \geq 1}$ and $\mathbb{M}$ be stochastic processes indexed by a metric space $\mathcal{H}$ and defined on some common probability space $(\Omega, \mathcal{F}, \mathbb{P})$. Consider a sequence $\left(\widehat{h}_{n}\right)_{n \geq 1}$, satisfying

$$
\mathbb{M}_{n}\left(\widehat{h}_{n}\right) \geq \sup _{h \in \mathcal{H}} \mathbb{M}_{n}(h)-\alpha_{n}
$$

where $\alpha_{n}=o_{\mathbb{P}}(1)$. Suppose that the following assumptions are fulfilled:

(i) $\mathbb{M}_{n} \rightsquigarrow \mathbb{M}$ in $\mathcal{L}^{\infty}(G)$ for every compact $G \subseteq \mathcal{H}$.

(ii) The trajectories of $\mathbb{M}$ are almost surely upper semicontinuous and posses a unique maximizer $\widehat{h}$, which is Borel measurable.

(iii) The sequence $\left(\widehat{h}_{n}\right)_{n \geq 1}$ is uniformly asymptotically tight, i.e. for every $\epsilon>0$, there is a compact set $G_{\epsilon} \subseteq \mathcal{H}$ such that

$$
\limsup _{n \rightarrow \infty} \mathbb{P}^{*}\left(\widehat{h}_{n} \notin G_{\epsilon}\right) \leq \epsilon,
$$

where $\mathbb{P}^{*}$ denotes the outer probability of $\mathbb{P}$. 
Then

$$
\widehat{h}_{n} \rightsquigarrow \widehat{h} \text { in } \mathcal{H} \text {. }
$$

Proof of Lemma A.1. In order to apply Theorem A.2 to Lemma A.1, we firstly show that the perturbation function $v$ is weakly lower semicontinuous on $\mathcal{P}_{0}(S)$. Since $\left\|\mathbf{Q}_{f}-\mathbf{Q}_{g}\right\|_{\mathbf{T V}}=\frac{1}{2} \| f-$ $g \|_{\mathcal{L}^{1}(S)}$, it is easy to see that $v$ is strongly convex with respect to the total variation norm. Now we show that the perturbation function $v$ is strongly convex with respect to the Prohorov metric. Fix two probability measures $\mathbb{P}, \mathbb{Q} \in \mathcal{P}(S)$ and $A \in \mathcal{B}(S)$. Let $\epsilon:=\|\mathbb{P}-\mathbb{Q}\|_{\mathrm{TV}}$. Then

$$
\begin{aligned}
\mathbb{P}(A) & \leq \mathbb{P}(A)-\mathbb{Q}(A)+\mathbb{Q}(A) \\
& \leq\|\mathbb{P}-\mathbb{Q}\|_{\mathbf{T V}}+\mathbb{Q}\left(A^{\epsilon}\right) \\
& =\mathbb{Q}\left(A^{\epsilon}\right)+\epsilon .
\end{aligned}
$$

Therefore by the definition of Prohorov metric, we have that $\rho(\mathbb{P}, \mathbb{Q}) \leq\|\mathbb{P}-\mathbb{Q}\|_{\text {TV }}$ for all $\mathbb{P}, \mathbb{Q} \in \mathcal{P}(S)$. This along with the fact that $v$ is strongly convex with respect to the total variation norm implies that $v$ is strongly convex with respect to the Prohorov metric. It then follows from Rockafellar (1970) that

$$
v(\widetilde{\mathbf{Q}}) \geq v(\mathbb{Q})+\int_{S} \nabla^{\mathbf{G}_{v}}(\mathbb{Q}) d(\widetilde{\mathbf{Q}}-\mathbf{Q})+\frac{L}{2} \rho(\widetilde{\mathbf{Q}}, \mathbf{Q})^{2}
$$

for all $\mathbb{Q}, \widetilde{\mathbf{Q}} \in \mathcal{P}_{0}(S)$. Now consider a sequence $\left(\mathbb{Q}_{n}\right)_{n \geq 1}, \mathbb{Q} \subset \mathcal{P}_{0}^{c}(S)$ such that $\rho\left(\mathbb{Q}_{n}, \mathbb{Q}\right) \rightarrow 0$. Setting $\widetilde{\mathbb{Q}}=\mathbb{Q}_{n}$ for $n \geq 1$ in Equation (16) we have

$$
v\left(\mathbb{Q}_{n}\right) \geq v(\mathbb{Q})+\int_{S} \nabla^{\mathbf{G}_{v}}(\mathbb{Q}) d\left(\mathbb{Q}_{n}-\mathbb{Q}\right)+\frac{L}{2} \rho\left(\mathbb{Q}_{n}, \mathbb{Q}\right)^{2}
$$

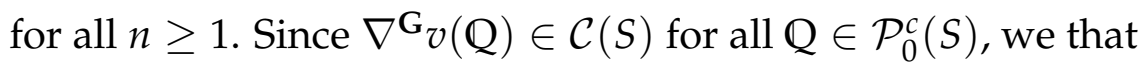

$$
\liminf _{n \rightarrow \infty} v\left(\mathbb{Q}_{n}\right) \geq v(\mathbb{Q})
$$

This proves that $v$ is weakly lower semicontinuous on $\mathcal{P}_{0}^{c}(S)$.

Consider the metric space $(\mathcal{P}(S), \rho)$. Since $S$ is compact, weak convergence of probability measures and convergence with respect to $\rho$ on the space $\mathcal{P}(S)$ are equivalent (see Billingsley (2013)). Let $\mathcal{O}(\mathcal{P}(S))$ denote the collection of all open sets in $\mathcal{P}(S)$. Let

$$
\mathrm{U}(\mathcal{P}(S)):=\{\psi: \mathcal{P}(S) \rightarrow \mathbb{R} \mid \psi \text { is weakly upper semicontinuous }\} .
$$


We consider the sup-vague topology on $\mathrm{U}(\mathcal{P}(S))$ (see Vervaat (1988) for details). The sets of the form

$$
\left\{\psi \in U(\mathcal{U}(S)) \mid \sup _{\mathbb{P} \in G} \psi(\mathbb{P})>x\right\}
$$

where $G$ varies over $\mathcal{O}(\mathcal{P}(S))$ and $x$ varies over $\mathbb{R}$ forms a base for the sup-vague topology on $\mathrm{U}(\mathcal{P}(S))$. Let $\mathcal{B}(\mathrm{U}(\mathcal{P}(S)))$ be the Borel sigma algebra on $\mathrm{U}(\mathcal{P}(S))$, that is, the smallest sigma algebra containing all open subsets of $\mathrm{U}(\mathcal{P}(S))$ with respect to the sup-vague topology.

For the rest of the proof, we assume that $\mathrm{U}(\mathcal{P}(S))$ is endowed with the Borel sigma algebra $\mathcal{B}(\mathrm{U}(\mathcal{P}(S))$ ), and $\mathcal{P}(S)$ is endowed with the Borel sigma algebra $\mathcal{B}(\mathcal{P}(S))$ generated by open sets of $\mathcal{P}(S)$.

Consider a sequence $\left(\mathbb{P}_{n}\right)_{n \geq 1}$ in $\mathcal{P}(S)$ such that $\rho\left(\mathbb{P}_{n}, \mathbb{P}\right) \rightarrow 0$. We need to show that $\rho\left(\mathcal{G}_{\eta}\left(\mathbb{P}_{n}\right), \mathcal{G}_{\eta}(\mathbb{P})\right) \rightarrow 0$. Fix a probability space $(\Omega, \mathcal{F}, \widehat{\lambda})$.

For every $n \geq 1$, let $\mathbb{M}_{n}: \Omega \rightarrow \mathrm{U}(\mathcal{P}(S))$ and $\widehat{h}_{n}: \Omega \rightarrow \mathcal{P}(S)$ be degenerate random variables, where $\mathbb{M}_{n}$ is indexed by $\mathcal{P}(S)$ and is defined as $\mathbb{M}_{n}(\mathbb{Q})=\int_{S} \pi\left(\mathbb{P}_{n}\right) d \mathbb{Q}-\eta v(\mathbb{Q})$ for all $\mathbb{Q} \in \mathcal{P}(S)$, and $\widehat{h}_{n}=\mathcal{G}_{\eta}\left(\mathbb{P}_{n}\right)$. Similarly, let $\mathbb{M}: \Omega \rightarrow \mathrm{U}(\mathcal{P}(S))$ and $\widehat{h}: \Omega \rightarrow \mathcal{P}(S)$ be degenerate random variables indexed by $\mathcal{P}(S)$ defined as $\mathbb{M}(\mathbb{Q})=\int_{S} \pi(\mathbb{P}) d \mathbb{Q}-\eta v(\mathbb{Q})$ for all $\mathbb{Q} \in \mathcal{P}(S)$ and $\widehat{h}=\mathcal{G}_{\eta}(\mathbb{P})$. By our formulation, $\left(\mathbb{M}_{n}\right)_{n \geq 1}$ and $\mathbb{M}$ are stochastic processes indexed by $\mathcal{P}(S)$ and are defined on the common probability space $(\Omega, \mathcal{F}, \widehat{\lambda})$. Moreover, by the definition of $\mathcal{G}_{\eta}\left(\mathbb{P}_{n}\right)$, we have that $\mathbb{M}_{n}\left(\mathcal{G}_{\eta}\left(\mathbb{P}_{n}\right)\right) \geq \sup _{\mathbb{Q} \in \mathcal{P}(S)} \mathbb{M}_{n}(\mathbb{Q})$ for all $n \geq 1$. We proceed to check Conditions (i), (ii), and (iii) in Theorem A.2.

Condition (i): Let $\epsilon>0$. Define the open ball of radius $\epsilon$ around $\mathbb{Q}$ as $B_{\epsilon}(\mathbb{Q}):=\{\mathbb{P} \in \mathcal{P}(S)$ : $\rho(\mathbb{Q}, \mathbb{P})<\epsilon\}$. We have

$$
\begin{aligned}
\left|\mathbb{M}_{n}(\mathbb{Q})-\mathbb{M}(\mathbb{Q})\right| & =\left|\int_{S}\left[\pi_{x}\left(\mathbb{P}_{n}\right)-\pi_{x}(\mathbb{P})\right] \mathbb{Q}(d x)\right| \\
& \leq \int_{S}\left|\pi_{x}\left(\mathbb{P}_{n}\right)-\pi_{x}(\mathbb{P})\right| \mathbb{Q}(d x)
\end{aligned}
$$

Now fix $\epsilon>0$. Then $\left(\mathrm{B}_{\epsilon}(\mathbb{Q})\right)_{\mathbb{Q} \in \mathcal{P}(S)}$ is an open cover of $\mathcal{P}(S)$. Note that since $(\mathcal{P}(S), \rho)$ is compact, there exists a $K_{\epsilon} \in \mathbb{N}$ and a finite subcollection $\left\{\mathbb{Q}_{1}^{\epsilon}, \ldots, \mathbb{Q}_{K_{\epsilon}}^{\epsilon}\right\}$ such that $\mathcal{P}(S) \subseteq \cup_{1 \leq i \leq K_{\epsilon}} \mathrm{B}_{\epsilon}\left(\mathbb{Q}_{i}^{\epsilon}\right)$. Let $\mathbb{Q} \in \mathcal{P}(S)$. Let $j \in\left\{1, \ldots, K_{\epsilon}\right\}$ be such that $\rho\left(\mathbb{Q}, \mathbb{Q}_{j}^{\epsilon}\right) \leq \epsilon$.

Putting these observations together, we have

$$
\begin{aligned}
\left\|\mathbb{M}_{n}-\mathbb{M}\right\|_{\infty} & =\sup _{\mathbb{Q} \in \mathcal{P}(S)}\left|\mathbb{M}_{n}(\mathbb{Q})-\mathbb{M}(\mathbb{Q})\right| \\
& \leq \max _{1 \leq i \leq K_{\epsilon}} \int_{S}\left|\pi_{x}\left(\mathbb{P}_{n}\right)-\pi_{x}(\mathbb{P})\right| \mathbb{Q}_{i}^{\epsilon}(d x)+\epsilon .
\end{aligned}
$$


By the assumption of the theorem, $\pi$ is a bounded and weakly continuous map. Therefore by Dominated Convergence Theorem, we have $\int_{S}\left|\pi_{x}\left(\mathbb{P}_{n}\right)-\pi_{x}(\mathbb{P})\right| \mathbb{Q}_{i}^{\epsilon}(d x) \rightarrow 0$ as $n \rightarrow \infty$ for all $1 \leq i \leq K_{\epsilon}$. So we have

$$
\begin{aligned}
\lim _{n \rightarrow \infty}\left\|\mathbb{M}_{n}-\mathbb{M}\right\|_{\infty} & \leq \lim _{n \rightarrow \infty} \max _{1 \leq i \leq K_{\epsilon}} \int_{S}\left|\pi_{x}\left(\mathbb{P}_{n}\right)-\pi_{x}(\mathbb{P})\right| \mathbb{Q}_{i}^{\epsilon}(d x)+\epsilon \\
& \rightarrow \epsilon .
\end{aligned}
$$

Since $\epsilon$ is arbitrary, $\mathbb{M}_{n}$ converges uniformly over $\mathcal{P}(S)$ (and therefore over every compact $G \subseteq \mathcal{P}(S))$ to $\mathbb{M}$. Since for all $\mathbb{Q} \in \mathcal{P}(S), \mathbb{M}_{n}(\mathbb{Q})$ is degenerate at $\int_{S} \pi\left(\mathbb{P}_{n}\right) d(\mathbb{Q})-\eta v(\mathbb{Q})$ for all $n \geq 1$, and $\mathbb{M}(\mathbb{Q})$ is degenerate at $\int_{S} \pi\left(\mathbb{P}_{n}\right) d(\mathbb{Q})-\eta v(\mathbb{Q})$, this implies that $\mathbb{M}_{n} \rightsquigarrow \mathbb{M}$ in $\mathcal{L}^{\infty}(G)$ for every compact $G \subseteq \mathcal{P}(S)$. This verifies Condition (i) of Theorem A.2.

Condition (ii): Since the perturbation function $v$ is lower semicontinuous with respect to $\rho$ (and appears with a minus sign in $\mathbb{M}$ ) and the mapping $\mathbb{Q} \mapsto \int_{S} \pi(\mathbb{P}) d \mathbb{Q}$ is continuous with respect to $\rho$, we have that $\mathbb{M}$ has almost surely upper semicontinuous paths on $\mathcal{P}_{0}^{c}(S)$ and a unique maximizer $\mathcal{G}_{\eta}(\mathbb{P}) \in \mathcal{P}_{0}^{c}(S)$. This verifies Condition (ii) of Theorem A.2 since Range $(\pi) \subseteq \mathcal{C}(S)$ and $v$ is admissible.

Condition (iii): Note that the space $(\mathcal{P}(S), \rho)$ is compact. Therefore, Condition (iii) holds if we choose $G_{\epsilon}=\mathcal{P}(S)$ for each $\epsilon>0$. This verifies Condition (iii) of Theorem A.2.

Now, we are ready to complete the proof of Lemma A.1. By Theorem A.2, we have $\widehat{h}_{n} \rightsquigarrow \widehat{h}$ in $\mathcal{P}(S)$. Since $\widehat{h}_{n}$ is degenerate at $\mathcal{G}_{\eta}\left(\mathbb{P}_{n}\right)$ for all $n \geq 1$, and $\widehat{h}$ is degenerate at $\mathcal{G}_{\eta}(\mathbb{P})$, it follows that $\rho\left(\mathcal{G}_{\eta}\left(\mathbb{P}_{n}\right), \mathcal{G}_{\eta}(\mathbb{P})\right) \rightarrow 0$. This completes the proof of Lemma A.1.

The proof of Theorem 3.1 is now complete by Theorem A.2 and Lemma A.1.

\section{A.2 Proof of TheOrem 3.2}

Let $\left(\eta_{n}\right)_{n \geq 1}$ be a sequence of positive perturbation parameters such that $\eta_{n} \downarrow 0$. Let $\mathbb{P}_{n}^{\circ}$ be a sequence of $\eta_{n}$-perturbed equilibrium for every $n \geq 1$. By compactness of $\mathcal{P}(S)$ under the weak topology, there exists a subsequence such that $\mathbb{P}_{n_{k}}^{\circ} \rightarrow^{w} \mathbb{P}^{\circ}$. We need to show that $\mathbb{P}^{\circ}$ is a Nash equilibrium of the original game. We prove this by contradiction. Suppose that $\mathbb{P}^{\circ}$ is not a Nash equilibrium. Then there exist $y \in S$ and $x \in \operatorname{supp}\left(\mathbb{P}^{\circ}\right)$, disjoint open balls $B_{y}$ and $B_{x}$, an $\epsilon>0$, 
and a large enough $N_{\epsilon}$ such that for all $n_{k} \geq N_{\epsilon}$, we have

$$
\inf _{z \in B_{y}} \pi_{z}\left(\mathbb{P}_{n_{k}}^{\circ}\right) \geq 2 \epsilon+\sup _{z \in B_{x}} \pi_{z}\left(\mathbb{P}_{n_{k}}^{\circ}\right)
$$

Weak convergence of $\mathbb{P}_{n_{k}}^{\circ}$ ensures the convergence of $\mathbb{P}_{n_{k}}^{\circ}\left(B_{x}\right)$. We show that $\mathbb{P}_{n_{k}}^{*}\left(B_{x}\right)$ converges to 0. First, we establish the result under Condition A1. Set $E=B_{x}, F=B_{y}$, and $g_{n_{k}}=\pi\left(\mathbb{P}_{n_{k}}^{\circ}\right)$. For $n_{k}>N_{\epsilon}$, it follows from (17) that $\inf _{s \in F} g_{n_{k}}(s)>\sup _{t \in E} g_{n_{k}}(t)$. Since $v$ satisfies Condition A1, it follows that for all $n_{k}>N_{\epsilon}$,

$$
\mathrm{D} \widetilde{v}_{\eta_{n_{k}}}^{*}\left(\pi\left(\mathbb{P}_{n_{k}}^{\circ}\right)\right)\left(\mathbb{I}_{B_{y}}\right) \geq u\left(\eta_{n_{k}}\right) \mathrm{D} \widetilde{v}_{\eta_{n_{k}}}^{*}\left(\pi\left(\mathbb{P}_{n_{k}}^{\circ}\right)\right)\left(\mathbb{I}_{B_{x}}\right)
$$

By Danskin's theorem, we have

$$
\mathrm{D} \widetilde{v}_{\eta_{n_{k}}}^{*}\left(\pi\left(\mathbb{P}_{n_{k}}^{\circ}\right)\right)\left(\mathbb{I}_{B_{y}}\right)=\left\langle\nabla^{\mathbf{F}} \widetilde{v}_{\eta_{n_{k}}}^{*}\left(\pi\left(\mathbb{P}_{n_{k}}^{\circ}\right)\right), \mathbb{I}_{B_{y}}\right\rangle
$$

Here, the dual pairing $\langle\cdot, \cdot\rangle: \mathcal{L}^{1}(S) \times \mathcal{L}^{\infty}(S) \rightarrow \mathbb{R}$ is defined as $\langle f, g\rangle:=\int_{S} f(x) g(x) d x$ for all $f \in \mathcal{L}^{1}(S)$ and all $g \in \mathcal{L}^{\infty}(S)$. Moreover,

$$
\begin{aligned}
\left\langle\nabla^{\mathbf{F}} \widetilde{v}_{\eta_{n_{k}}}^{*}\left(\pi\left(\mathbb{P}_{n_{k}}^{\circ}\right)\right), \mathbb{I}_{B_{y}}\right\rangle & =\int_{S} \nabla^{\mathbf{F}} \widetilde{v}_{\eta_{n_{k}}^{*}}\left(\pi\left(\mathbb{P}_{n_{k}}^{\circ}\right)\right)(t) \mathbb{I}_{B_{y}}(t) d t \\
& =\int_{B_{y}} \nabla^{\mathbf{F}} \widetilde{v}_{\eta_{n_{k}}}^{*}\left(\pi\left(\mathbb{P}_{n_{k}}^{\circ}\right)\right)(t) d t \\
& =\xi\left(\nabla^{\mathbf{F}} \widetilde{v}_{\eta_{n_{k}}}\left(\pi\left(\mathbb{P}_{n_{k}}^{\circ}\right)\right)\right)\left(B_{y}\right) \\
& =\mathbb{P}_{n_{k}}^{\circ}\left(B_{y}\right) .
\end{aligned}
$$

Combining all these observations, we have $\widetilde{D}_{\eta_{n_{k}}}^{*}\left(\pi\left(\mathbb{P}_{n_{k}}^{\circ}\right)\right)\left(\mathbb{I}_{B_{y}}\right)=\mathbb{P}_{n_{k}}^{\circ}\left(B_{y}\right)$. Similarly, we can obtain D $\widetilde{v}_{\eta_{n_{k}}}^{*}\left(\pi\left(\mathbb{P}_{n_{k}}^{\circ}\right)\right)\left(\mathbb{I}_{B_{x}}\right)=\mathbb{P}_{n_{k}}^{\circ}\left(B_{x}\right)$. Therefore, (18) implies $\mathbb{P}_{n_{k}}^{\circ}\left(B_{y}\right) \geq u\left(\eta_{n_{k}}\right) \mathbb{P}_{n_{k}}^{\circ}\left(B_{x}\right)$ for $n_{k}>N_{\epsilon}$. Since $u\left(\eta_{n_{k}}\right) \rightarrow \infty$ as $k \rightarrow \infty$, this implies that $\mathbb{P}_{\eta_{n_{k}}}^{\circ}\left(B_{x}\right) \rightarrow 0$. Moreover, since $\mathbb{P}_{n_{k}}^{\circ} \rightarrow^{w} \mathbb{P}^{\circ}$, we have by Portmanteau's theorem that $\mathbb{P}^{\circ}\left(B_{x}\right)=0$. However, this leads to a contradiction since $x \in \operatorname{supp}\left(\mathbb{P}^{\circ}\right)$. Therefore $\mathbb{P}^{\circ}$ must be a Nash equilibrium of the original game.

Next, we establish the result under Condition A2. Continuing from (17), we once again show by contradiction that $\mathbb{P}_{n_{k}}^{\circ}\left(B_{x}\right) \rightarrow 0$. Suppose that $\mathbb{P}_{n_{k}}^{\circ}\left(B_{x}\right) \rightarrow c$, where $c>0$. This implies that $\mathbb{P}_{n_{k}}^{\circ}\left(B_{x}\right) \geq \frac{c}{2}$ for large $k$ such that $n_{k} \geq N^{\prime}$ (say). For every $k \geq 1$, let $\widetilde{\mathbb{P}}_{n_{k}}^{\circ}$ be a shift of $\mathbb{P}_{n_{k}}^{\circ}$ from $B_{x}$ 
to $B_{y}$. Then by some algebra we have that

$$
\int_{B_{x} \cup B_{y}} \pi_{z}\left(\mathbb{P}_{n_{k}}^{\circ}\right) \widetilde{\mathbb{P}}_{n_{k}}^{\circ}(d z) \geq 2 \epsilon\left(\mathbb{P}_{n_{k}}^{\circ}\left(B_{x}\right)-\widetilde{\mathbb{P}}_{n_{k}}^{\circ}\left(B_{x}\right)\right)+\int_{B_{x} \cup B_{y}} \pi_{z}\left(\mathbb{P}_{n_{k}}^{\circ}\right) \mathbb{P}_{n_{k}}^{\circ}(d z)
$$

for all $n_{k} \geq \max \left\{N_{\epsilon}, N^{\prime}\right\}$. Since $\eta_{n} \downarrow 0$ and $v$ satisfies Condition (A2), there exists $N^{\prime \prime}$ such that for all $n_{k} \geq N^{\prime \prime}$, we have

$$
\begin{aligned}
\left|v_{\eta_{n_{k}}}\left(\widetilde{\mathbb{P}}_{n_{k}}^{\circ}\right)-v_{\eta_{n_{k}}}\left(\mathbb{P}_{n_{k}}^{\circ}\right)\right| & =\left|\eta_{n_{k}}\right|\left|v\left(\widetilde{\mathbb{P}}_{n_{k}}^{\circ}\right)-v\left(\mathbb{P}_{n_{k}}^{\circ}\right)\right| \\
& \leq \epsilon\left(\mathbb{P}_{n_{k}}^{\circ}\left(B_{x}\right)-\widetilde{\mathbb{P}}_{n_{k}}^{\circ}\left(B_{x}\right)\right)
\end{aligned}
$$

for $n_{k} \geq \max \left\{N_{\epsilon}, N^{\prime}, N^{\prime \prime}\right\}$. This in turn implies that

$$
v_{\eta_{n_{k}}}\left(\widetilde{\mathbb{P}}_{n_{k}}^{\circ}\right) \leq v_{\eta_{n_{k}}}\left(\mathbb{P}_{n_{k}}^{\circ}\right)+\epsilon\left(\mathbb{P}_{n_{k}}^{\circ}\left(B_{x}\right)-\widetilde{\mathbb{P}}_{n_{k}}^{\circ}\left(B_{x}\right)\right)
$$

for $n_{k} \geq \max \left\{N_{\epsilon}, N^{\prime}, N^{\prime \prime}\right\}$. Note that

$$
\int_{S \backslash\left(B_{x} \cup B_{y}\right)} \pi_{z}\left(\mathbb{P}_{n_{k}}^{\circ}\right) \mathbb{P}_{n_{k}}^{\circ}(d z)=\int_{S \backslash\left(B_{x} \cup B_{y}\right)} \pi_{z}\left(\mathbb{P}_{n_{k}}^{\circ} \widetilde{\mathbb{P}}_{n_{k}}^{\circ}(d z)\right.
$$

for all $k \geq 1$. Therefore we have that

$$
\int_{S} \pi_{z}\left(\mathbb{P}_{n_{k}}^{\circ}\right) \widetilde{\mathbb{P}}_{n_{k}}^{\circ}(d z)-\eta_{n_{k}} v\left(\widetilde{\mathbb{P}}_{n_{k}}^{\circ}\right) \geq \epsilon\left(\mathbb{P}_{n_{k}}^{\circ}\left(B_{x}\right)-\widetilde{\mathbb{P}}_{n_{k}}^{\circ}\left(B_{x}\right)\right)+\int_{S} \pi_{z}\left(\mathbb{P}_{n_{k}}^{\circ}\right) \mathbb{P}_{n_{k}}^{\circ}(d z)-\eta_{n_{k}} v\left(\mathbb{P}_{n_{k}}^{\circ}\right)
$$

for large enough $k$, contradicting the fact that $\mathbb{P}_{n_{k}}^{\circ}$ is a $\eta_{n_{k}}$-perturbed equilibrium for every $n \geq 1$. Therefore $\mathbb{P}_{n_{k}}^{\circ}\left(B_{x}\right) \rightarrow 0$. Since $\mathbb{P}_{n_{k}}^{\circ} \rightarrow \mathbb{P}^{\circ}$, we must have that $\mathbb{P}^{\circ}\left(B_{x}\right)=0$, which is a contradiction, since $x$ is in the support of $\mathbb{P}^{\circ}$. Therefore $\mathbb{P}^{\circ}$ is a Nash equilibrium of the original game. This concludes the proof of Theorem 3.2.

\section{A.3 TSALlis ENTROPY AND BURG ENTROPY}

We show that both the Tsallis $(\gamma$ ) entropy, $0<\gamma<1$ (Example 3.2), and the Burg entropy (Example 3.3) satisfy Condition A2 with respect to the sequence $\left(\mathbb{P}_{n_{k}}^{\circ}\right)_{k \geq 1}$, where $\left(\mathbb{P}_{n_{k}}^{\circ}\right)_{k \geq 1}$ is as defined in the proof of Theorem 3.1. To show this, consider two disjoint subsets $E, F \subseteq S$ with $\mathbb{P}_{n_{k}}^{\circ}(E) \nrightarrow 0$ with $\inf _{z \in F} \pi_{z}\left(\mathbb{P}_{n_{k}}^{\circ}\right)>\sup _{w \in E} \pi_{w}\left(\mathbb{P}_{n_{k}}^{\circ}\right)$ for sufficiently large $k$. Since $\left(\mathbb{P}_{n_{k}}^{\circ}\right)_{k \geq 1} \subseteq \mathcal{P}_{0}^{c}(S)$, it follows that there exists $\left(A_{n_{k}}\right)_{k \geq 1} \subseteq E$ such that $p_{n_{k}}^{\circ}$ is bounded away from 0 uniformly of $A_{n_{k}}$ for sufficiently large $k$, in which case there exist a sequence of probability measures $\left(\widetilde{\mathbb{P}}_{n_{k}}^{\circ}\right)_{k \geq 1}$, where $\widetilde{\mathbb{P}}_{n_{k}}^{\circ}$ is a shift 
of $\mathbb{P}_{n_{k}}^{\circ}$ from $A_{n_{k}}$ to $F$ for all $k \geq 1$ (Definition 3.6).

(i) Tsallis $(\gamma)$ entropy satisfies Condition A2: By the definition of shifted probability measures (Definition 3.6), for every $k \geq 1$, the probability density function of $\widetilde{\mathbb{P}}_{n_{k}}^{\circ}$ is the same as the density of $\mathbb{P}_{n_{k}}^{\circ}$ on the set $S \backslash\left(A_{n_{k}} \cup F\right)$. Therefore it is enough to bound the difference in entropy on $A_{n_{k}} \cup F$. Note that

$$
\left|v^{\mathbf{t}}\left(\widetilde{\mathbb{P}}_{n_{k}}^{\circ}\right)-v^{\mathbf{t}}\left(\mathbb{P}_{n_{k}}^{\circ}\right)\right|=\left|\int_{A_{n_{k}} \cup F} \frac{\widetilde{p}_{n_{k}}^{\circ}(x)-\widetilde{p}_{n_{k}}^{\circ}(x)^{\gamma}}{\gamma(1-\gamma)} d x-\int_{A_{n_{k}} \cup F} \frac{p_{n_{k}}^{\circ}(x)-p_{n_{k}}^{\circ}(x)^{\gamma}}{\gamma(1-\gamma)} d x\right|
$$

where $\widetilde{p}_{n_{k}}^{\circ}$ and $p_{n_{k}}^{\circ}$ denote the probability densities of $\widetilde{\mathbb{P}}_{n_{k}}^{\circ}$ and $\mathbb{P}_{n_{k^{\prime}}}^{\circ}$, respectively, for every $k \geq 1$.

By Definition 3.6, we have that

$$
\widetilde{p}_{n_{k}}^{\circ}(x)=\alpha_{n_{k}} p_{n_{k}}^{\circ}(x) \quad \text { for all } x \in F
$$

where $\alpha_{n_{k}}=1+\frac{\mathbb{P}_{n_{k}}^{\circ}\left(A_{n_{k}}\right)-\widetilde{\mathbb{P}}_{n_{k}}^{\circ}\left(A_{n_{k}}\right)}{\mathbb{P}_{n_{k}}^{\circ}(F)}$ for all $k \geq 1$. Since $\alpha_{n_{k}}>1$ for all $k \geq 1$ and $0<\gamma<1$, we have that $\alpha_{n_{k}}^{\gamma}-1<\alpha_{n_{k}}-1$ for all $k \geq 1$. Therefore, we have in particular

$$
\alpha_{n_{k}}^{\gamma}-1<\frac{\mathbb{P}_{n_{k}}^{\circ}\left(A_{n_{k}}\right)-\widetilde{\mathbb{P}}_{n_{k}}^{\circ}\left(A_{n_{k}}\right)}{\mathbb{P}_{n_{k}}^{\circ}(F)} \quad \text { for all } k \geq 1
$$

First we bound the difference in the entropy on the ball $F$. We have

$$
\begin{aligned}
& \int_{F}\left|\frac{\widetilde{p}_{n_{k}}^{\circ}(x)-\widetilde{p}_{n_{k}}^{\circ}(x)^{\gamma}}{\gamma(1-\gamma)}-\frac{p_{n_{k}}^{\circ}(x)-p_{n_{k}}^{\circ}(x)^{\gamma}}{\gamma(1-\gamma)}\right| d x \\
& \leq \int_{F}\left|\frac{\widetilde{p}_{n_{k}}^{\circ}(x)-p_{n_{k}}^{\circ}(x)}{\gamma(1-\gamma)}\right| d x+\int_{F}\left|\frac{\widetilde{p}_{n_{k}}^{\circ}(x)^{\gamma}-p_{n_{k}}^{\circ}(x)^{\gamma}}{\gamma(1-\gamma)}\right| d x \\
& \leq \frac{1}{\gamma(1-\gamma)}\left[\left(\alpha_{n_{k}}-1\right) \int_{F} p_{n_{k}}^{\circ}(x) d x+\left(\alpha_{n_{k}}^{\gamma}-1\right) \int_{F} p_{n_{k}}^{\circ}(x)^{\gamma} d x\right] \\
& \leq \frac{1}{\gamma(1-\gamma)}\left[\left(\mathbb{P}_{n_{k}}^{\circ}\left(A_{n_{k}}\right)-\widetilde{\mathbb{P}}_{n_{k}}^{\circ}\left(A_{n_{k}}\right)\right)+\frac{\left(\mathbb{P}_{n_{k}}^{\circ}\left(A_{n_{k}}\right)-\widetilde{\mathbb{P}}_{n_{k}}^{\circ}\left(A_{n_{k}}\right)\right)}{\mathbb{P}_{n_{k}}^{\circ}(F)}\left(\int_{B_{y}} p_{n_{k}}^{\circ}(x) d x\right)^{\gamma}\right] \\
& \leq \frac{\left(\mathbb{P}_{n_{k}}^{\circ}\left(A_{n_{k}}\right)-\widetilde{\mathbb{P}}_{n_{k}}^{\circ}\left(A_{n_{k}}\right)\right)}{\gamma(1-\gamma)}\left[1+\frac{1}{\mathbb{P}_{n_{k}}^{\circ}(F)}\right] .
\end{aligned}
$$

Note that the second last inequality is due to Jensen's inequaity since the mapping $t \mapsto t^{\gamma}$ is concave for $0<\gamma<1$. We now show that $\mathbb{P}_{n_{k}}^{\circ}(F)$ is bounded away from 0 . Since $\mathbb{P}_{n_{k}}^{\circ}$ is a 
Nash equilibrium for every $k \geq 1$, it follows from Héliou et al. (2020) that

$$
\mathbb{P}_{n_{k}}^{\circ}(A)=\left[\frac{\eta_{n_{k}}}{\gamma(1-\gamma)}\right]^{1 /(1-\gamma)} \int_{A} \frac{1}{\left(\theta_{n_{k}}-\pi_{z}\left(\mathbb{P}_{n_{k}}^{\circ}\right)\right)^{1 /(1-\gamma)}} d z
$$

for all $A \in \mathcal{B}(S)$, where for every $k \geq 1$, we choose $\theta_{n_{k}}>\left\|\pi\left(\mathbb{P}_{n_{k}}^{\circ}\right)\right\|_{\infty}$ such that

$$
\left[\frac{\eta_{n_{k}}}{\gamma(1-\gamma)}\right]^{1 /(1-\gamma)} \int_{S} \frac{1}{\left(\theta_{n_{k}}-\pi_{z}\left(\mathbb{P}_{n_{k}}^{\circ}\right)\right)^{1 /(1-\gamma)}} d z=1
$$

Letting $\epsilon \rightarrow 0$ in (17), we have that

$$
\int_{F} \frac{1}{\left(\theta_{n_{k}}-\pi_{z}\left(\mathbb{P}_{n_{k}}^{\circ}\right)\right)^{1 /(1-\gamma)}} d z \geq \int_{E} \frac{1}{\left(\theta_{n_{k}}-\pi_{z}\left(\mathbb{P}_{n_{k}}^{\circ}\right)\right)^{1 /(1-\gamma)}} d z
$$

for all $k \geq 1$. Therefore we have that $\mathbb{P}_{n_{k}}^{\circ}(F) \geq \mathbb{P}_{n_{k}}^{\circ}(E)$ for all $k \geq 1$. Since $\mathbb{P}_{n_{k}}^{\circ}\left(B_{x}\right)$ is bounded away from 0 , we have that $\mathbb{P}_{n_{k}}^{\circ}(F)$ is also bounded away from 0 . That is, there exists $c>0$ such that $\mathbb{P}_{n_{k}}^{\circ}(F) \geq c$ for all $k \geq 1$. Therefore in particular we have

$$
\int_{F}\left|\frac{\widetilde{p}_{n_{k}}^{\circ}(x)-\widetilde{p}_{n_{k}}^{\circ}(x)^{\gamma}}{\gamma(1-\gamma)}-\frac{p_{n_{k}}^{\circ}(x)-p_{n_{k}}^{\circ}(x)^{\gamma}}{\gamma(1-\gamma)}\right| d x \leq \frac{(c+1)\left(\mathbb{P}_{n_{k}}^{\circ}\left(A_{n_{k}}\right)-\widetilde{\mathbb{P}}_{n_{k}}^{\circ}\left(A_{n_{k}}\right)\right)}{c \gamma(1-\gamma)} .
$$

Now, it follows that

$$
\begin{aligned}
\int_{A_{n_{k}}}\left|\frac{\widetilde{p}_{n_{k}}^{\circ}(x)-\widetilde{p}_{n_{k}}^{\circ}(x)^{\gamma}}{\gamma(1-\gamma)}-\frac{p_{n_{k}}^{\circ}(x)-p_{n_{k}}^{\circ}(x)^{\gamma}}{\gamma(1-\gamma)}\right| d x \leq \frac{1}{\gamma(1-\gamma)}\left[\mathbb{P}_{n_{k}}^{\circ}\left(A_{n_{k}}\right)-\widetilde{\mathbb{P}}_{n_{k}}^{\circ}\left(A_{n_{k}}\right)\right. & \left.+\int_{A_{n_{k}}}\left|p_{n_{k}}^{\circ}(x)^{\gamma}-\widetilde{p}_{n_{k}}^{\circ}(x)^{\gamma}\right| d t\right] .
\end{aligned}
$$

It now follows that there exists a constant $\widetilde{c}>0$ such that for sufficiently large $k$, we have $p_{n_{k}}^{\circ}(x) \geq \widetilde{c}$ for all $x \in A_{n_{k}}$. Therefore, for sufficiently large $k$ we have that the mapping $t \mapsto t^{\gamma}$ is Lipschitz on $A_{n_{k}}$ in which case we have that

$$
\int_{A_{n_{k}}}\left|p_{n_{k}}^{\circ}(x)^{\gamma}-\widetilde{p}_{n_{k}}^{\circ}(x)^{\gamma}\right| d t \leq \frac{\gamma}{\widetilde{c}^{1-\gamma}}\left(\mathbb{P}_{n_{k}}^{\circ}\left(A_{n_{k}}\right)-\widetilde{\mathbb{P}}_{n_{k}}^{\circ}\left(A_{n_{k}}\right)\right)
$$

for sufficiently large $k$. Therefore in particular we have that

$$
\int_{A_{n_{k}}}\left|\frac{\widetilde{p}_{n_{k}}^{\circ}(x)-\widetilde{p}_{n_{k}}^{\circ}(x)^{\gamma}}{\gamma(1-\gamma)}-\frac{p_{n_{k}}^{\circ}(x)-p_{n_{k}}^{\circ}(x)^{\gamma}}{\gamma(1-\gamma)}\right| d x \leq \frac{\left(1+\frac{\gamma}{\widetilde{c}^{1-\gamma}}\right)\left(\mathbb{P}_{n_{k}}^{\circ}\left(A_{n_{k}}\right)-\widetilde{\mathbb{P}}_{n_{k}}^{\circ}\left(A_{n_{k}}\right)\right)}{\gamma(1-\gamma)}
$$


Finally, for sufficiently large $k$, we have that

$$
\left|v_{\eta}^{\mathbf{t}}\left(\widetilde{\mathbb{P}}_{n_{k}}^{\circ}\right)-v_{\eta}^{\mathbf{t}}\left(\mathbb{P}_{n_{k}}^{\circ}\right)\right| \leq \frac{\eta\left(2 c+\frac{\gamma}{\widetilde{c}^{1-\gamma}}+1\right)\left(\mathbb{P}_{n_{k}}^{\circ}\left(A_{n_{k}}\right)-\widetilde{\mathbb{P}}_{n_{k}}^{\circ}\left(A_{n_{k}}\right)\right)}{c \gamma(1-\gamma)}
$$

Since $E, F \subseteq S$ are arbitrary, it follows that Tsallis entropy satisfies Condition A2.

(ii) Burg entropy satisfies Condition A2: As in the previous case, we have that the densities of $\mathbb{P}_{n_{k}}^{\circ}$ and $\widetilde{\mathbb{P}}_{n_{k}}^{\circ}$ remain unchanged on $S \backslash\left(A_{n_{k}} \cup F\right)$ for every $k \geq 1$. Therefore it is enough to bound the difference in entropy on $A_{n_{k}} \cup F$. Note that,

$$
\left|v^{\mathbf{b}}\left(\widetilde{\mathbb{P}}_{n_{k}}^{\circ}\right)-v^{\mathbf{b}}\left(\mathbb{P}_{n_{k}}^{\circ}\right)\right|=\left|\int_{A_{n_{k}} \cup F} \log \widetilde{p}_{n_{k}}^{\circ}(x) d x-\int_{A_{n_{k}} \cup F} \log p_{n_{k}}^{\circ}(x) d x\right|
$$

Observe that

$$
\widetilde{p}_{n_{k}}^{\circ}(x)=\alpha_{n_{k}} p_{n_{k}}^{\circ}(x) \quad \text { for all } x \in F,
$$

where $\alpha_{n_{k}}=1+\frac{\mathbb{P}_{n_{k}}^{\circ}\left(A_{n_{k}}\right)-\widetilde{\mathbb{P}}_{n_{k}}^{\circ}\left(A_{n_{k}}\right)}{\mathbb{P}_{n_{k}}^{\circ}(F)}$ for all $k \geq 1$. Since $\log (1+x)<x$ for all $x>0$, we have that $\log \alpha_{n_{k}}<\frac{\mathbb{P}_{n_{k}}^{\circ}\left(A_{n_{k}}\right)-\widetilde{\mathbb{P}}_{n_{k}}^{\circ}\left(A_{n_{k}}\right)}{\mathbb{P}_{n_{k}}^{\circ}(F)}$ for all $k \geq 1$. Therefore, we have

$$
\begin{aligned}
& \int_{F}\left|\log \widetilde{p}_{n_{k}}^{\circ}(x)-\log p_{n_{k}}^{\circ}(x)\right| d x=\int_{F}\left|\log \alpha_{n_{k}} p_{n_{k}}^{\circ}(x)-\log p_{n_{k}}^{\circ}(x)\right| d x \\
& =\int_{F}\left|\log \alpha_{n_{k}}\right| d x \\
& =\int_{F}\left|\log \left(1+\frac{\mathbb{P}_{n_{k}}^{\circ}\left(A_{n_{k}}\right)-\widetilde{\mathbb{P}}_{n_{k}}^{\circ}\left(A_{n_{k}}\right)}{\mathbb{P}_{n_{k}}^{\circ}(F)}\right)\right| d x \\
& \leq \frac{\lambda(F)}{\mathbb{P}_{n_{k}}^{\circ}(F)}\left(\mathbb{P}_{n_{k}}^{\circ}\left(A_{n_{k}}\right)-\widetilde{\mathbb{P}}_{n_{k}}^{\circ}\left(A_{n_{k}}\right)\right) \text {. }
\end{aligned}
$$

Since $\mathbb{P}_{n_{k}}^{\circ}(E)$ is bounded away from 0 for sufficiently large $k$, we have by similar arguments that $\mathbb{P}_{n_{k}}^{\circ}(F)$ is bounded away from 0 for sufficiently large $k$. In other words, $\mathbb{P}_{n_{k}}^{\circ}(F) \geq c$ for sufficiently large $k$. Therefore we have

$$
\left.\int_{F}\left|\log \widetilde{p}_{n_{k}}^{\circ}(x)-\log p_{n_{k}}^{\circ}(x)\right| d x \leq \frac{\lambda(F)}{c}\left(\mathbb{P}_{n_{k}}^{\circ} A_{n_{k}}\right)-\widetilde{\mathbb{P}}_{n_{k}}^{\circ}\left(A_{n_{k}}\right)\right)
$$

for sufficiently large $k$. By similar arguments, as in the case of Tsallis entropy, there exists $\bar{c}>0$ such that that $p_{n_{k}}^{\circ}(x) \geq \bar{c}$ for all $t \in A_{n_{k}}$ for sufficiently large $k$. Therefore, for 
sufficiently large $k$, the mapping $t \mapsto \log t$ is Lipschitz of $A_{n_{k}}$. This implies that

$$
\int_{A_{n_{k}}}\left|\log \widetilde{p}_{n_{k}}^{\circ}(x)-\log p_{n_{k}}^{\circ}(x)\right| \leq \frac{1}{\bar{c}}\left(\mathbb{P}_{n_{k}}^{\circ}\left(A_{n_{k}}\right)-\widetilde{\mathbb{P}}_{n_{k}}^{\circ}\left(A_{n_{k}}\right)\right)
$$

for sufficiently large $k$. So we have

$$
\left|v_{\eta}^{\mathbf{b}}\left(\widetilde{\mathbb{P}}_{n_{k}}^{\circ}\right)-v_{\eta}^{\mathbf{b}}\left(\mathbb{P}_{n_{k}}^{\circ}\right)\right| \leq \eta\left(\frac{\lambda(F)}{c}+\frac{1}{\bar{c}}\right)\left(\mathbb{P}_{n_{k}}^{\circ}\left(A_{n_{k}}\right)-\widetilde{\mathbb{P}}_{n_{k}}^{\circ}\left(A_{n_{k}}\right)\right)
$$

for sufficiently large $k$. Since $E, F \subseteq S$ are arbitrary, it follows that Burg entropy satisfies Condition A2.

\section{A.4 Appendix to Section 4}

\section{A.4.1 ProOF OF LeMma 4.1}

Recall the definitions of $\mathcal{L}_{+}^{1,1}(S)$ in $(1)$ and $\mathcal{L}_{+, D}^{1,1}(S)$ in (11). Let $\xi$ be the bijective map $\xi: \mathcal{L}_{+}^{1,1}(S) \rightarrow$ $\mathcal{P}_{0}(S)$ defined by $\xi(f)=\mathbb{Q}_{f}$, where $\mathbb{Q}_{f}$ is as defined immediately following (1). Note that the restriction of $\xi$ to $\mathcal{L}_{+, D}^{1,1}(S)$ is a bijection between $\mathcal{L}_{+, D}^{1,1}(S)$ and $\mathcal{P}_{D}(S)$ as defined in (10).

Without loss of generality, it is enough to prove the lemma for $\eta=1$. Let $\pi: \mathcal{P}(S) \rightarrow \mathcal{L}^{\infty}(S)$ and let $\nabla^{\mathbf{F}} \widetilde{v}^{*}(g) \in \mathcal{L}_{+, D}^{1,1}(S)$ for all $g \in \mathcal{L}^{\infty}(S)$. We show that $\Delta_{D}$ is forward invariant under the generalized dynamic, that is, $\mathcal{G}_{1}(\mathbb{P}) \in \mathcal{P}_{D}(S)$ for all $\mathbb{P} \in \mathcal{P}_{D}(S)$. Since $\mathcal{L}^{1}(S)^{*} \cong \mathcal{L}^{\infty}(S)$ (see Rudin (2006)), we have $\widetilde{v}^{*}(g)=\sup _{f \in \mathcal{L}^{1}(S)}[\langle f, g\rangle-\widetilde{v}(f)]$ for all $g \in \mathcal{L}^{\infty}(S)$, where $\langle f, g\rangle:=$ $\int_{S} f(x) g(x) d x$. Since $\widetilde{v}$ is closed and $L$-strong convex, we have for all $g \in \mathcal{L}^{\infty}(S)$ (see Rockafellar (1970) for details),

$$
\nabla^{\mathbf{F}} \widetilde{v}^{*}(g)=\arg \max _{f \in \mathcal{L}^{1}(S)}[\langle f, g\rangle-\widetilde{v}(f)]
$$

Since $\widetilde{v}(f)=\infty$ for all $f \in \mathcal{L}^{1}(S) \backslash \mathcal{L}_{+}^{1,1}(S)$ we have

$$
\arg \max _{f \in \mathcal{L}^{1}(S)}[\langle f, g\rangle-\widetilde{v}(f)]=\arg \max _{f \in \mathcal{L}_{+}^{1,1}(S)}[\langle f, g\rangle-\widetilde{v}(f)]
$$

Therefore,

$$
\begin{aligned}
\arg \max _{f \in \mathcal{L}^{1}(S)}[\langle f, g\rangle-\widetilde{v}(f)] & =\arg \max _{f \in \mathcal{L}_{+}^{1,1}(S)}[\langle f, g\rangle-\widetilde{v}(f)] \\
& =\arg \max _{f \in \mathcal{L}_{+}^{1,1}(S)}\left(\int_{S} f(x) g(x) \mathrm{d} x-\widetilde{v}(f)\right)
\end{aligned}
$$




$$
\begin{aligned}
& =\arg \max _{f \in \mathcal{L}_{+}^{1,1}(S)}\left(\int_{S} g(x) f(x) \mathrm{d} x-\widetilde{v}(f)\right) \\
& =\arg \max _{f \in \mathcal{L}_{+}^{1,1}(S)}\left(\int_{S} g \mathrm{dQ}_{f}-v\left(\mathbb{Q}_{f}\right)\right) .
\end{aligned}
$$

This implies

$$
\xi\left(\arg \max _{f \in \mathcal{L}_{+}^{1,1}(S)}[\langle f, g\rangle-\widetilde{v}(f)]\right)=\arg \max _{\mathbf{Q}_{f} \in \mathcal{P}_{0}(S)}\left[\int_{S} g \mathrm{~d} \mathbb{Q}_{f}-v\left(\mathbb{Q}_{f}\right)\right]
$$

By the assumption of the lemma, $\nabla^{\mathbf{F}} \widetilde{v}^{*}(g) \in \mathcal{L}_{+, D}^{1,1}(S)$ for all $g \in \mathcal{L}^{\infty}(S)$. Fix $\mathbb{P} \in \mathcal{P}_{D}(S)$. Taking $g=\pi(\mathbb{P})$ and using (21) we have

$$
\begin{aligned}
\mathcal{G}_{1}(\mathbb{P}) & =\arg \max _{\mathbf{Q} g \in \mathcal{P}_{0}(S)}\left[\int_{S} \pi(\mathbb{P}) \mathrm{dQ}_{g}-v\left(\mathbb{Q}_{g}\right)\right] \\
& =\xi\left(\arg \max _{f \in \mathcal{L}_{+}^{1,1}(S)}[\langle f, \pi(\mathbb{P})\rangle-\widetilde{v}(f)]\right) \\
& =\xi\left(\nabla^{\mathbf{F}} \widetilde{v}^{*}(\pi(\mathbb{P}))\right) \in \mathcal{P}_{D}(S) .
\end{aligned}
$$

This concludes our proof.

\section{A.4.2 Proof of Theorem 4.1}

We use the generalized Picard-Lindelöf Theorem to prove Theorem 4.1.

Theorem A.3 (The Generalized Picard-Lindelöf Theorem, Zeidler (1986)). Let Y be a Banach space. Let $y_{0} \in Y, t_{0} \in \mathbb{R}$, and

$$
Q_{b}:=\left\{(t, y) \in \mathbb{R} \times Y|| t-t_{0} \mid \leq a \text { and }\left\|y-y_{0}\right\| \leq b\right\}
$$

for fixed $a, b>0$. Suppose $f: Q_{b} \rightarrow Y$ is continuous and that

$$
\begin{gathered}
\|f(t, x)-f(t, y)\| \leq L\|x-y\| \text { for all }(t, x),(t, y) \in Q_{b}, \text { and } \\
\qquad\|f(t, x)\|<K \text { for all }(t, x) \in Q_{b}
\end{gathered}
$$

where $L \geq 0$ and $K>0$ are fixed. Choose $c$ such that $0<c<a$ and $K c<b$. Then the initial value problem

$$
\dot{x}(t)=f(t, x(t)), \quad x\left(t_{0}\right)=y_{0},
$$


where $x:\left[t_{0}-c, t_{0}+c\right] \rightarrow Y$, has exactly one continuously differetiable solution on the interval $\left[t_{0}-c, t_{0}+c\right]$.

By Theorem A.3 it is enough to show that the mapping $\mathbb{P} \mapsto \mathcal{G}_{\eta}(\mathbb{P})$ is Lipschitz with respect to the total variation norm. We show this in the following lemma.

Lemma A.2. The mapping $\mathbb{P} \mapsto \mathcal{G}_{\eta}(\mathbb{P})-\mathbb{P}$ is Lipschitz continuous with respect to the total variation norm.

Proof. By Definition 4.1, we need to show that

$$
\left\|\mathcal{G}_{\eta}(\mathbb{P})-\mathcal{G}_{\eta}(\widetilde{\mathbb{P}})\right\|_{\mathbf{T V}} \leq\|\mathbb{P}-\widetilde{\mathbb{P}}\|_{\mathbf{T V}}
$$

for all $\mathbb{P}, \widetilde{\mathbb{P}} \in \mathcal{P}(S)$. Consider the mapping $\xi:\left(\mathcal{L}_{+}^{1,1}(S),\|\cdot\|_{\mathcal{L}^{1}(S)}\right) \rightarrow\left(\mathcal{P}_{0}(S),\|\cdot\|_{\mathrm{TV}}\right)$. We show that $\xi$ is 1-Lipschitz. For $f, g \in \mathcal{L}_{+}^{1,1}(S)$, we have

$$
\begin{aligned}
\|\xi(f)-\xi(g)\|_{\mathbf{T V}} & =\left\|\mathbf{Q}_{f}-\mathbf{Q}_{g}\right\|_{\mathbf{T V}} \\
& =\frac{1}{2} \int_{S}|f(x)-g(x)| d x \\
& \leq\|f-g\|_{\mathcal{L}^{1}(S)} .
\end{aligned}
$$

We complete the proof in two steps: first we prove that the mapping $\mathbb{P} \mapsto \mathcal{G}_{\eta}(\mathbb{P})$ is Lipschitz with respect to the total variation norm, and second, we use this to prove the same for $\mathbb{P} \mapsto$ $\mathcal{G}_{\eta}(\mathbb{P})-\mathbb{P}$. Since $\pi$ is $\beta$-Lipschitz, we have $\|\pi(\mathbb{P})-\pi(\widetilde{\mathbb{P}})\|_{\infty} \leq \beta\|\mathbb{P}-\widetilde{\mathbb{P}}\|_{\text {TV }}$ for all $\mathbb{P}, \widetilde{\mathbb{P}} \in \mathcal{P}(S)$. Let $\widetilde{v}_{\eta}(f):=\eta \widetilde{v}(f)$ for all $f \in \mathcal{L}^{1}(S)$. Since $\widetilde{v}$ is closed and strongly convex, $\eta \widetilde{v}$ is closed and $\eta L$-strongly convex. Therefore, $\left\|\nabla^{\mathbf{F}} \widetilde{v}_{\eta}^{*}(g)-\nabla^{\mathbf{F}} \widetilde{v}_{\eta}^{*}(\widetilde{g})\right\|_{\infty} \leq \frac{1}{\eta L}\|g-\widetilde{g}\|_{\infty}$ for all $g, \widetilde{g} \in \mathcal{P}(S)$ (see Rockafellar (1970), Theorem 23.5). Therefore it follows from Lemma 4.1 that

$$
\begin{aligned}
\left\|\mathcal{G}_{\eta}(\mathbb{P})-\mathcal{G}_{\eta}(\widetilde{\mathbb{P}})\right\|_{\mathbf{T V}} & =\left\|\xi\left(\nabla^{\mathbf{F}} \widetilde{v}_{\eta}^{*}(\pi(\mathbb{P}))\right)-\xi\left(\nabla^{\mathbf{F}} \widetilde{v}_{\eta}^{*}(\pi(\widetilde{\mathbb{P}}))\right)\right\|_{\mathbf{T V}} \\
& \leq\left\|\nabla^{\mathbf{F}} \widetilde{v}_{\eta}^{*}(\pi(\mathbb{P}))-\nabla^{\mathbf{F}} \widetilde{v}_{\eta}^{*}(\pi(\widetilde{\mathbb{P}}))\right\|_{\mathcal{L}^{1}(S)} \\
& \leq \frac{1}{\eta L}\|\pi(\mathbb{P})-\pi(\widetilde{\mathbb{P}})\|_{\infty} \\
& \leq \frac{\beta}{\eta L}\|\mathbb{P}-\widetilde{\mathbb{P}}\|_{\mathbf{T V}}
\end{aligned}
$$

Finally,

$$
\left\|\left(\mathcal{G}_{\eta}(\mathbb{P})-\mathbb{P}\right)-\left(\mathcal{G}_{\eta}(\widetilde{\mathbb{P}})-\widetilde{\mathbb{P}}\right)\right\|_{\mathbf{T V}} \leq\left\|\mathcal{G}_{\eta}(\mathbb{P})-\mathcal{G}_{\eta}(\widetilde{\mathbb{P}})\right\|_{\mathbf{T V}}+\|\mathbb{P}-\widetilde{\mathbb{P}}\|_{\mathbf{T V}}
$$




$$
\leq\left(\frac{\beta}{\eta L}+1\right)\|\mathbb{P}-\widetilde{\mathbb{P}}\|_{\mathbf{T V}}
$$

This completes the proof of Lemma A.2.

The proof of Lemma A.2 establishes one part of Theorem 4.1, the existence of a unique solution from every initial state. To prove the continuity of the semiflow (Definition 4.2) of the dynamic, we apply Gronwall's Lemma (see Zeidler (1986) for details) which we state below.

Lemma A.3 (Zeidler (1986)). Let $u, v:\left[t_{0}-a, t_{0}+a\right] \rightarrow V$ be maps into the subspace $V$ of a Banach space $Y$. Suppose $f:\left[t_{0}-a, t_{0}+a\right] \times V \rightarrow Y$ is continuous and Lipschitz continuous with respect to $u$, i.e.,

$$
\|f(t, u)-f(t, v)\| \leq L\|u-v\| \text { for all } t \in\left[t_{0}-a, t_{0}+a\right] \text { and } u, v \in V \text { and fixed } L>0
$$

Then $\|u(t)-v(t)\| \leq e^{L\left|t-t_{0}\right|}\|u(0)-v(0)\|$ for all $t \in\left[t_{0}-a, t_{0}+a\right]$.

We apply Lemma A.3 with $Y=\mathcal{M}(S), V=\mathcal{P}(S)$ and $f=\mathcal{G}_{\eta}$. By Lemma A.2, the conditions of Lemma A.3 are satisfied. Therefore, there exists $k>0$ such that for any two solutions $\left(\mathbb{P}_{t}\right)_{t \geq 0}$ and $\left(\mathbb{Q}_{t}\right)_{t \geq 0}$ to the Generalized Dynamic with initial conditions $\mathbb{P}_{0}$ and $\mathbb{Q}_{0}$, respectively, we have $\left\|\mathbb{P}_{t}-\mathbb{Q}_{t}\right\|_{\mathbf{T V}} \leq e^{k t}\left\|\mathbb{P}_{0}-\mathbb{Q}_{0}\right\|_{\mathbf{T V}}$. This establishes continuity of the semiflow of the Generalized Dynamic. This concludes the proof of Theorem 4.1 .

\section{A.5 Appendix to SECTION 5}

We first prove Lemma 5.1. For that, we require another lemma.

Lemma A.4. Suppose $\Phi: \mathcal{M}(S) \rightarrow \mathbb{R}$ is the perturbed expected payoff in a population game $\pi$. That is $\Phi(\mu)=\int_{S} \pi(\mathbb{P}) d \mu-v(\mu)$ where v satisfies the properties as stated before. Then there exists a unique maximizer $\mu^{*}$ such that

$$
d v(\widehat{\mu})(v)=\int_{S} \pi(\mathbb{P}) d v
$$

Proof. The fact $v$ is strongly convex and lower semicontinuous imply that there exists an unique maximizer of $\Phi$, say $\widehat{\mu}$. Then,

$$
\begin{aligned}
0 & =\mathrm{d} \Phi(\widehat{\mu})(v) \\
& =\lim _{\epsilon \rightarrow 0} \frac{\Phi(\widehat{\mu}+\epsilon \nu)-\Phi(\widehat{\mu})}{\epsilon}
\end{aligned}
$$




$$
\begin{aligned}
& =\lim _{\epsilon \rightarrow 0} \frac{\epsilon \int_{S} \pi(\mathbb{P}) d v-v(\widehat{\mu}+\epsilon v)+v(\widehat{\mu})}{\epsilon} \\
& =\int_{S} \pi(\mathbb{P}) d v-\mathrm{d} v(\widehat{\mu}) v .
\end{aligned}
$$

Therefore we must have $\mathrm{d} v(\widehat{\mu})(v)=\int_{S} \pi(\mathbb{P}) d v$.

Proof of Lemma 5.1. We need to show that $\int_{S} \widetilde{\pi}_{x}(\mathbb{P}) \dot{\mathbb{P}}(d x) \geq 0$ for all $\mathbb{P} \in \mathcal{P}(S)$. In other words, we need to show that $\int_{S} \tilde{\pi}_{x}(\mathbb{P})\left(\mathcal{G}_{\eta}(\mathbb{P})-\mathbb{P}\right)(d x) \geq 0$. By Lemma A.4 we have $\int_{S} \pi(\mathbb{P}) d \mathbb{P}=$

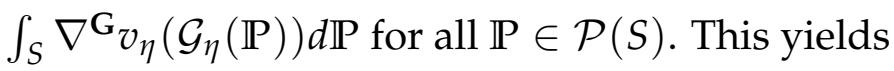

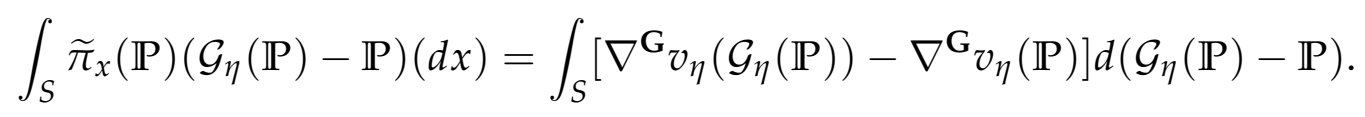

Since $v_{\eta}$ is strongly convex (and hence convex), it follows (see Rockafellar (1970)) that $v_{\eta}(\widetilde{\mathbb{Q}}) \geq$ $v_{\eta}(\mathbf{Q})+\int_{S} \nabla v_{\eta}(\mathbf{Q}) d(\widetilde{\mathbf{Q}}-\mathbf{Q})$ for all $\widetilde{\mathbf{Q}}, \mathbf{Q} \in \mathcal{P}(S)$. Setting $\widetilde{\mathbb{Q}}=\mathcal{G}_{\eta}(\mathbb{P})$ and $\mathbb{Q}=\mathbb{P}$, and interchanging the roles of $\widetilde{\mathbb{Q}}$ and $\mathbb{Q}$, we obtain

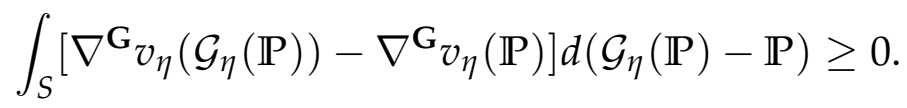

It remains to show that equality holds in (23) only if $\mathcal{G}_{\eta}(\mathbb{P})=\mathbb{P}$, (i.e, $\dot{\mathbb{P}}=0$ ). We first show that

$$
\mathrm{d} v_{\eta}(\mathbb{P}) \mu=\int_{S} \Theta^{\prime} \circ p(x) \mu(d x)
$$

for all $\mathbb{P} \in \mathcal{P}_{0}(S)$ and all $\mu \in \mathcal{M}_{0}(S)$. Fix $\mathbb{P} \in \mathcal{P}_{0}(S)$ and $\mu \in \mathcal{M}_{0}(S)$ with density functions $p$ and $\mu_{0}$ respectively. Since the perturbation function satisfies (13), it follows that for every $\epsilon>0$

$$
v_{\eta}(\mathbb{P}+\epsilon \mu)=\int_{S} \Theta \circ\left(p+\mu_{0}\right)(x) d x=\int_{S} \Theta \circ p(x) d x+\epsilon \mu_{0}(x) \Theta^{\prime} \circ p(x) d x+\mathbf{o}\left(\epsilon^{2}\right) .
$$

Therefore (24) follows that Equation (25) and Definition 3.1. It now follows that $\nabla^{\mathbf{G}_{v_{\eta}}}(\mathbb{P})=\Theta^{\prime} \circ p$ for all $\mathbb{P} \in \mathcal{P}_{0}(S)$.

We now proceed to prove part (i) of Lemma 5.1. Let $p_{\eta}$ denote the density function of $\mathcal{G}_{\eta}(\mathbb{P})$.

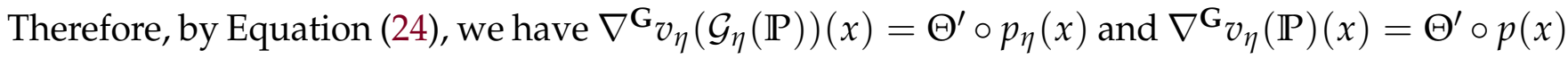
for all $x \in S$. This in particular means that

$$
\int_{S}\left[\nabla^{\mathbf{G}_{v_{\eta}}}\left(\mathcal{G}_{\eta}(\mathbb{P})\right)-\nabla^{\mathbf{G}} v_{\eta}(\mathbb{P})\right] d\left(\mathcal{G}_{\eta}(\mathbb{P})-\mathbb{P}\right)=\int_{S}\left(\Theta^{\prime} \circ p_{\eta}(x)-\Theta^{\prime} \circ p(x)\right)\left(p_{\eta}(x)-p(x)\right) d x .
$$

By assumption of part (i) the lemma, $\Theta$ is either strictly increasing or strictly decreasing on $[0, \infty)$. 
Therefore the integrand in the RHS Equation (26) is either non negative or non positive for all $x \in S$. This implies that equality holds in Equation (26) if and only if $p_{\eta}(x)=p(x)$ for all $x \in S$. This proves part (i) of Lemmma 5.1.

We now prove part (ii) of Lemma 5.1. It follows from Lemma 4.1 that for every $\eta>0$, $\mathcal{G}_{\eta}(\mathbb{P}) \in \mathcal{P}_{D_{\eta}}(S)$ for every $\mathbb{P} \in \mathcal{P}(S)$. This in particular means that $D_{\eta}^{-1} \leq p_{\eta}(x) \leq D_{\eta}$ for all $x \in S$. By assumption of part (ii) of the lemma, there exists $0 \leq \alpha<1<\beta$ such that $\Theta$ is either strictly increasing or strictly decreasing on $[\alpha, \beta]$.

Let $\eta>0$ be such that $\left[D_{\eta}^{-1}, D_{\eta}\right] \subset[\alpha, \beta]$. It then follows that

$$
\left(\Theta^{\prime} \circ p_{\eta}(x)-\Theta^{\prime} \circ p(x)\right)\left(p_{\eta}(x)-p(x)\right)
$$

is either non negative or non positive for all $x \in S$ and all $\mathbb{P} \in \mathcal{P}_{D_{\eta}}(S)$ according as $\Theta$ is strictly increasing or decreasing on $[\alpha, \beta]$. This implies that

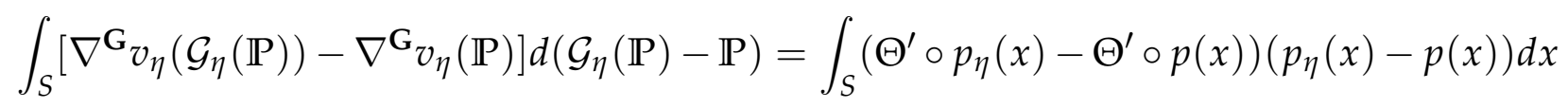

is either non negative or non positive according as $\Theta$ is strictly increasing or decreasing on $[\alpha, \beta]$, with equality if and only if $p_{\eta}(x)=p(x)$ for all $x \in S$. This proves part (ii) of Lemma 5.1.

This concludes the proof of Lemma 5.1.

Proof of Theorem 5.1. Let $\left\{\mathbb{P}_{t}\right\}_{t \geq 0}$ be the solution trajectory with initial condition $\mathbb{P}_{0} \in \mathcal{P}_{D}(S)$. We have already shown that $\mathcal{P}_{D}(S)$ is forward invariant under the generalized dynamic. This implies that $\mathbb{P}_{t} \in \mathcal{P}_{D}(S)$ for all $t>0$ whenever $\mathbb{P}_{0} \in \mathcal{P}_{D}(S)$. Note that

$$
\begin{aligned}
& \dot{\tilde{\varphi}}(\mathbb{P})=\dot{\varphi}(\mathbb{P})-\dot{v}_{\eta}(\mathbb{P}) \\
& =\mathrm{D} \varphi(\mathbb{P}) \dot{\mathbb{P}}-\mathrm{d} v_{\eta}(\mathbb{P}) \dot{\mathbb{P}}
\end{aligned}
$$

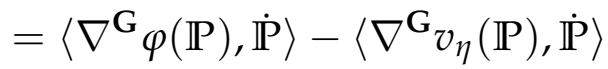

$$
\begin{aligned}
& =\langle\pi(\mathbb{P}), \dot{\mathbb{P}}\rangle-\left\langle\nabla^{\mathrm{G}} v_{\eta}(\mathbb{P}), \dot{\mathbb{P}}\right\rangle \\
& =\int_{S} \pi(\mathbb{P}) \mathrm{d} \dot{\mathbb{P}}-\int_{S} \nabla^{\mathbf{G}_{v_{\eta}}}(\mathbb{P}) \mathrm{d} \dot{\mathbb{P}}
\end{aligned}
$$

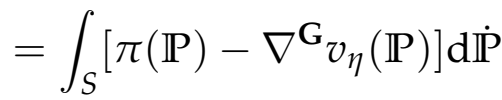

$$
\begin{aligned}
& =\int_{S} \tilde{\pi}(\mathbb{P}) \mathrm{d} \dot{\mathbb{P}} \geq 0 \text {. }
\end{aligned}
$$

The last inequality follows from Lemma 5.1. Hence $\Lambda\left(\mathbb{P}_{t}\right)$ increases strictly whenever $\mathbb{P}_{0}$ is not the rest point of the generalized dynamic. 
We know that the space $\mathcal{P}_{D}(S)$ is compact with respect to the strong (total variation) topology. The fact that the vector field in the generalized dynamic is Lipschitz, a continuous and unique solution exists starting from $\mathbb{P}_{0} \in \mathcal{P}_{D}(S)$. Form standard results of dynamical systems theory (see Bhatia and Szegö (2006)) it follows that the $\omega$-limit set of the trajectory $\left\{\mathbb{P}_{t}\right\}_{t \geq 0}$ is non-empty compact and connected in the strong topology. Moreover this set is contained in the set consisting of the rest points of the dynamic. Hence any element in the $\omega$-limit set is a rest point of the generalized dynamic and hence a generalized equilibrium. Also since the entropy adjusted potential function is increasing along every solution trajectory, any point in the $\omega$-limit set will be a maximizer of the function. This concludes the proof of Theorem 5.1.

\section{A.6 Appendix to Section 6}

Proof of Lemma 6.1. We prove the lemma along the lines of Lahkar and Riedel (2015). Since the generalized dynamic is forward invariant on $\mathcal{P}_{D}(S)$, it suffices to prove uniqueness on $\mathcal{P}_{D}(S)$. Fix $\mathbb{P} \in \mathcal{P}_{D}(S)$ and consider the virtual payoff

$$
\tilde{\pi}(\mathbb{P})=\pi(\mathbb{P})-\nabla^{\mathbf{G}_{v_{\eta}}(\mathbb{P})}
$$

for every $\mathbb{P} \in \mathcal{P}(S)$. Then for every $\mu \in \mathcal{M}_{0}(S)$ and $t \in \mathbb{R}$, such that $\mathbb{P}+t \mu \in \mathcal{P}_{D}(S)$, define

$$
\varrho(\mathbb{P}, \mu, t):=\int_{S} \tilde{\pi}(\mathbb{P}+t \mu) \mu(d x)
$$

We now compute the Gâteaux derivative of $\varrho(\mathbb{P}, \mu,$.$) with respect to t$. By similar arguments as in Lahkar and Riedel (2015), we obtain

$$
\frac{\partial \varrho(\mathbb{P}, \mu, t)}{\partial t}=\int_{S} \mathrm{~d} \widetilde{\pi}_{x}(\mathbb{P}+t \mu) \mu(d x)
$$

We now compute integrand on the right hand side of the above equation. Note that by definition of $\tilde{\pi}$, we have

$$
(\mathrm{d} \tilde{\pi}(\mathbb{P}+t \mu) \mu)(x)=(\mathrm{d} \pi(\mathbb{P}+t \mu) \mu)(x)-\mathrm{d} \nabla^{\mathbf{G}_{v_{\eta}}(\mathbb{P}+t \mu) \mu(x) .}
$$

Therefore integrating both sides of the above equation we have,

$$
\int_{S} \mathrm{~d} \widetilde{\pi}_{x}(\mathbb{P}+t \mu) \mu(d x)=\int_{S} \mathrm{~d} \pi_{x}(\mathbb{P}+t \mu) \mu(d x)-\int_{S} \mathrm{~d} \nabla^{\mathrm{G}_{v_{\eta}}}(\mathbb{P}+t \mu) \mu(d x) .
$$


Since $\pi$ is a Fréchet differentiable, the Gâteaux derivative of $\pi$ coincides with the Fréchet derivative of $\pi$. Also since negative semidefinite game, we have

$$
\int_{S} \mathrm{~d} \pi_{x}(\mathbb{P}+t \mu) \mu(d x)=\int_{S} \mathrm{D} \pi_{x}(\mathbb{P}+t \mu) \mu(d x) \leq 0
$$

By assumptions of the lemma, we have

$$
\int_{S} \mathrm{~d} \nabla^{\mathrm{G}} v_{\eta}(\mathbb{P}+\epsilon \mu) \mu(d x) \geq 0
$$

This in turn implies that

$$
\frac{\partial \varrho(\mathbb{P}, \mu, t)}{\partial t}<0
$$

The proof now follows along the lines of Lahkar and Riedel (2015). This completes the proof of Theorem 6.1.

Proof of Theorem 6.1. We first define some notations which we require in the proof. Let $\Lambda_{D}^{1}(\mathbb{P}):=$ $\int_{S} \pi_{x}(\mathbb{P}) \mathcal{G}_{\eta}(\mathbb{P})(d x), \quad \Lambda_{D}^{2}(\mathbb{P}):=v_{\eta}\left(\mathcal{G}_{\eta}(\mathbb{P})\right), \quad \Lambda_{D}^{3}(\mathbb{P}):=\int_{S} \pi_{x}(\mathbb{P}) \mathbb{P}(d x)$ and $\Lambda_{D}^{4}(\mathbb{P}):=v_{\eta}(\mathbb{P})$ for all $\mathbb{P} \in \mathcal{P}_{0}(S)$. Since Gâtaeux derivative is linear, it is enough to compute the Gâtaeux derivative of $\Lambda_{D}^{i}(\mathbb{P})$ for $i=1, \ldots, 4$ separately. Differentiating $\Lambda_{D}^{1}$ we have

$$
\begin{aligned}
\mathrm{D} \Lambda_{D}^{1}(\mathbb{P}) \dot{\mathbb{P}} & =\int_{S} \mathrm{D} \pi(\mathbb{P}) \dot{\mathbb{P}}(x) \mathcal{G}_{\eta}(\mathbb{P})(d x)+\int_{S} \pi_{x}(\mathbb{P}) \dot{\mathcal{G}_{\eta}}(\mathbb{P})(d x) \\
& =\left\langle\mathrm{D} \pi(\mathbb{P}) \dot{\mathbb{P}}, \mathcal{G}_{\eta}(\mathbb{P})\right\rangle+\left\langle\pi(\mathbb{P}), \dot{\mathcal{G}}_{\eta}(\mathbb{P})\right\rangle
\end{aligned}
$$

Since the perturbation function $v$ is Gâteaux differentiable, we have

$$
\begin{aligned}
\mathrm{d} \Lambda_{D}^{2}(\mathbb{P}) \dot{\mathbb{P}} & =\mathrm{d} v_{\eta}\left(\mathcal{G}_{\eta}(\mathbb{P})\right) \dot{\mathcal{G}_{\eta}}(\mathbb{P}) \\
& =\left\langle\nabla^{\mathrm{G}_{v_{\eta}}}\left(\mathcal{G}_{\eta}(\mathbb{P})\right), \dot{\mathcal{G}}_{\eta}(\mathbb{P})\right\rangle
\end{aligned}
$$

Now, differentiating $\Lambda_{D}^{3}$ we have

$$
\begin{aligned}
\mathrm{D} \Lambda_{D}^{3}(\mathbb{P}) \dot{\mathbb{P}} & =\int_{S} \pi_{x}(\mathbb{P}) \dot{\mathbb{P}} \mathbb{P}(d x)+\int_{S} \pi_{x}(\mathbb{P}) \dot{\mathbb{P}}(d x) \\
& =\langle\mathrm{D} \pi(\mathbb{P}) \dot{\mathbb{P}}, \mathbb{P}\rangle+\langle\pi(\mathbb{P}), \dot{\mathbb{P}}\rangle
\end{aligned}
$$


Finally differentiating we have $\Lambda_{D}^{4}$ we have

$$
\begin{aligned}
\mathrm{d} \Lambda_{D}^{4}(\mathbb{P}) \dot{\mathbb{P}} & =\mathrm{d} v_{\eta}(\mathbb{P}) \dot{\mathbb{P}} \\
& =\left\langle\nabla^{\mathrm{G}} v_{\eta}(\mathbb{P}), \dot{\mathbb{P}}\right\rangle
\end{aligned}
$$

Combining all the observations from above we have

$$
\begin{aligned}
& \dot{\Lambda}_{D}(\mathbb{P})=\left\langle\mathrm{D} \pi(\mathbb{P}) \dot{\mathbb{P}}, \mathcal{G}_{\eta}(\mathbb{P})\right\rangle+\left\langle\pi(\mathbb{P}), \dot{\mathcal{G}}_{\eta}(\mathbb{P})\right\rangle-\left\langle\nabla^{\mathrm{G}_{v_{\eta}}}\left(\mathcal{G}_{\eta}(\mathbb{P})\right), \dot{\mathcal{G}}_{\eta}(\mathbb{P})\right\rangle \\
& -\langle\mathrm{D} \pi(\mathbb{P}) \dot{\mathbb{P}}, \mathbb{P}\rangle-\langle\pi(\mathbb{P}), \dot{\mathbb{P}}\rangle+\left\langle\nabla^{\mathrm{G}_{v_{\eta}}}(\mathbb{P}), \dot{\mathbb{P}}\right\rangle . \\
& =\left\langle\mathrm{D} \pi(\mathbb{P}) \dot{\mathbb{P}}, \mathcal{G}_{\eta}(\mathbb{P})-\mathbb{P}\right\rangle+\left\langle\pi(\mathbb{P}), \dot{\mathcal{G}_{\eta}}(\mathbb{P})-\dot{\mathbb{P}}\right\rangle+\left\langle\nabla^{\mathrm{G}_{v_{\eta}}}(\mathbb{P}), \dot{\mathbb{P}}\right\rangle-\left\langle\nabla^{\mathrm{G}_{v_{\eta}}}\left(\mathcal{G}_{\eta}(\mathbb{P})\right), \dot{\mathcal{G}}_{\eta}(\mathbb{P})\right\rangle .
\end{aligned}
$$

Since $\pi$ is a negative semidefinite game and $\dot{\mathbb{P}}=\mathcal{G}_{\eta}(\mathbb{P})-\mathbb{P} \in \mathcal{M}_{0}(S)$, we have from the definition of negative semidefinite game that the first term in the RHS on the above equation, namely $\left\langle\mathrm{D} \pi(\mathbb{P}) \dot{\mathbb{P}}, \mathcal{G}_{\eta}(\mathbb{P})-\mathbb{P}\right\rangle \leq 0$. Using this inequality in the RHS of the above expression and rearranging the terms we have

$$
\dot{\Lambda}_{D}(\mathbb{P}) \leq\left\langle\pi(\mathbb{P})-\nabla^{G_{v_{\eta}}}\left(\mathcal{G}_{\eta}(\mathbb{P})\right), \dot{\mathcal{G}}_{\eta}(\mathbb{P})\right\rangle-\left\langle\pi(\mathbb{P})-\nabla^{\mathrm{G}_{v_{\eta}}}(\mathbb{P}), \dot{\mathbb{P}}\right\rangle
$$

Observe that the first term on the right is 0 due to Lemma A.4 and the second term on the right is non negative due to Lemma 5.1. Therefore $\dot{\Lambda}_{D}(\mathbb{P}) \leq 0$ along every solution which originated in $\mathcal{P}_{D}(\mathrm{~S})$. This in particular means the Lyapunov function as defined in (15) decrease along every solution trajectory which originates in $\mathcal{P}_{D}(S)$. Since $\mathcal{P}_{D}(S)$ is compact under the strong topology and $\dot{\Lambda}_{D}(\mathbb{P}) \leq 0$ with equality only from the rest points, it follows from standard results of dynamical systems theory (Bhatia and Szegö (2006)) implies that $\omega$-limit of every solution trajectory satisfies $\dot{\Lambda}_{D}(\mathbb{P})=0$, which is the set of all rest points of the GPBR dynamic. In particular, we have from Lemma 6.1 every non stationary solution originating in $\mathcal{P}_{D}(S)$ converges to the unique generalized perturbed equilibrium.

\section{REFERENCES}

[1] Michel Benaim and Morris W Hirsch. Mixed equilibria and dynamical systems arising from fictitious play in perturbed games. Games and Economic Behavior, 29(1-2):36-72, 1999.

[2] Nam P Bhatia and George P Szegö. Dynamical systems: stability theory and applications, volume 35. Springer, 2006. 
[3] Patrick Billingsley. Convergence of probability measures. John Wiley \& Sons, 2013.

[4] D Charalambos and Border Aliprantis. Infinite Dimensional Analysis: A Hitchhiker's Guide. Springer-Verlag Berlin and Heidelberg GmbH \& Company KG, 2013.

[5] Man-Wah Cheung. Pairwise comparison dynamics for games with continuous strategy space. Journal of Economic Theory, 153:344-375, 2014.

[6] Man-Wah Cheung. Imitative dynamics for games with continuous strategy space. Games and Economic Behavior, 99:206-223, 2016.

[7] Man-Wah Cheung and Ratul Lahkar. Nonatomic potential games: the continuous strategy case. Games and Economic Behavior, 108:341-362, 2018.

[8] Dietmar Ferger. A continuous mapping theorem for the argmax-functional in the non-unique case. Statistica Neerlandica, 58(1):83-96, 2004.

[9] Drew Fudenberg and David K. Levine. The theory of learning in games, volume 2. MIT press, 1998.

[10] Itzhak Gilboa and Akihiko Matsui. Social stability and equilibrium. Econometrica: Journal of the Econometric Society, pages 859-867, 1991.

[11] Harald Hanche-Olsen and Helge Holden. The kolmogorov-riesz compactness theorem. Expositiones Mathematicae, 28(4):385-394, 2010.

[12] Amélie Héliou, Matthieu Martin, Panayotis Mertikopoulos, and Thibaud Rahier. Online non-convex optimization with imperfect feedback. arXiv preprint arXiv:2010.08496, 2020.

[13] Josef Hofbauer. Stability for the best response dynamics. preprint, 1995.

[14] Josef Hofbauer and Ed Hopkins. Learning in perturbed asymmetric games. Games and Economic Behavior, 52(1):133-152, 2005.

[15] Josef Hofbauer and William H Sandholm. On the global convergence of stochastic fictitious play. Econometrica, 70(6):2265-2294, 2002.

[16] Josef Hofbauer and William H Sandholm. Evolution in games with randomly disturbed payoffs. Journal of economic theory, 132(1):47-69, 2007.

[17] Josef Hofbauer and William H Sandholm. Stable games and their dynamics. Journal of Economic theory, 144(4):1665-1693, 2009.

[18] Josef Hofbauer, Jörg Oechssler, and Frank Riedel. Brown-von neumann-nash dynamics: the continuous strategy case. Games and Economic Behavior, 65(2):406-429, 2009.

[19] Ratul Lahkar. Convergence to walrasian equilibrium with minimal information. Journal of Economic Interaction and Coordination, 15(3):553-578, 2020.

[20] Ratul Lahkar and Saptarshi Mukherjee. Evolutionary implementation in a public goods 
game. Journal of Economic Theory, 181:423-460, 2019.

[21] Ratul Lahkar and Saptarshi Mukherjee. Evolutionary implementation in aggregative games. Mathematical Social Sciences, 109:137 - 151, 2021. ISSN 0165-4896. doi: https://doi.org/10. 1016/j.mathsocsci.2020.11.004.

[22] Ratul Lahkar and Frank Riedel. The logit dynamic for games with continuous strategy sets. Games and Economic Behavior, 91:268-282, 2015.

[23] Lars-Göran Mattsson and Jörgen W Weibull. Probabilistic choice and procedurally bounded rationality. Games and Economic Behavior, 41(1):61-78, 2002.

[24] John Maynard Smith. Evolution and the Theory of Games. Cambridge university press, 1982.

[25] Dov Monderer and Lloyd S Shapley. Potential games. Games and economic behavior, 14(1): 124-143, 1996.

[26] Jörg Oechssler and Frank Riedel. Evolutionary dynamics on infinite strategy spaces. Economic Theory, 17(1):141-162, 2001.

[27] Jörg Oechssler and Frank Riedel. On the dynamic foundation of evolutionary stability in continuous models. Journal of Economic Theory, 107(2):223-252, 2002.

[28] Steven Perkins and David S Leslie. Stochastic fictitious play with continuous action sets. Journal of Economic Theory, 152:179-213, 2014.

[29] R Tyrrell Rockafellar. Convex analysis. Number 28. Princeton university press, 1970.

[30] Walter Rudin. Real and complex analysis. Tata McGraw-hill education, 2006.

[31] William H Sandholm. Potential games with continuous player sets. Journal of Economic theory, 97(1):81-108, 2001.

[32] William H Sandholm. Population games and evolutionary dynamics. MIT press, 2010.

[33] AN Shiryaev. Probability 2nd edition springer-verlag isbn 0-387-94549-0 new york usa pp 1-621. 315. Shiryaev AN 1998a Foundations of stochastic financial mathematics, 1:1-492, 1995.

[34] Constantino Tsallis. Possible generalization of boltzmann-gibbs statistics. Journal of statistical physics, 52(1-2):479-487, 1988.

[35] Aad W Van Der Vaart and Jon A Wellner. Weak convergence. In Weak convergence and empirical processes, pages 16-28. Springer, 1996.

[36] Wim Vervaat. Random upper semicontinuous functions and extremal processes. Department of Mathematical Statistics, (R 8801), 1988.

[37] E Zeidler. Nonlinear functional analysis and its applications, i springer. New York, NY, 1986. 